\title{
Stability and Convergence Analysis of a Class of Continuous Piecewise Polynomial Approximations for Time-Fractional Differential Equations
}

\author{
Han Zhou' ${ }^{1} \cdot$ Paul Andries Zegeling ${ }^{1}$
}

Received: 9 August 2017 / Revised: 26 January 2018 / Accepted: 20 March 2018 /

Published online: 2 May 2018

(C) The Author(s) 2018

\begin{abstract}
We propose and study a class of numerical schemes to approximate time-fractional differential equations. The methods are based on the approximations of the Caputo fractional derivative of order $\alpha \in(0,1)$ by using continuous piecewise polynomials, which are strongly related to the backward differentiation formulae. We investigate their theoretical properties, such as the local truncation error and global error estimates with respect to sufficiently smooth solutions, and the numerical stability in terms of stability region and $A\left(\frac{\pi}{2}\right)$-stability. Numerical experiments are given to verify our theoretical investigations.
\end{abstract}

Keywords Caputo fractional derivative - Continuous piecewise polynomial - Time-fractional differential equations $\cdot$ Stability $\cdot$ Convergence analysis

\section{Introduction}

Fractional calculus, as a generalization of classical calculus, has been an intriguing topic for many famous mathematicians since the end of the 17th century. During the last 4 decades, many scholars have been working on the development of theory for fractional derivatives and integrals, found their ways in the world of fractional calculus and their applications. For more detailed information on the historical background, we refer the interested reader to the following books: $[6,21,22,34-36,38]$ and [20]. As an application of fractional calculus, differential equations possessing terms with fractional derivatives in the space- or time- or space-time direction have become very important in many areas. Particularly, in recent years a huge amount of interesting and surprising fractional models have been proposed. Here, we mention just a few typical applications: in the theory of Hankel transforms [15], in financial

\footnotetext{
$凶 \quad$ Han Zhou

zhhmath@qq.com

Paul Andries Zegeling

p.a.zegeling@uu.nl

1 Department of Mathematics, Utrecht University, Utrecht, The Netherlands
} 
models [40,42], in elasticity theory [5], in medical applications [23,39], in geology [8,27], in physics $[7,10,33]$ and many more.

Similar to the work for ordinary differential equations, investigation of numerical methods for time-fractional differential equations (tfDEs) started its development. This paper will consider numerical approaches to tfDEs of the form

$$
{ }^{C} D^{\alpha} u(t)=f(t, u(t)), \quad t \in(0, T]
$$

with initial condition $u(0)=u_{0}$, where the operator ${ }^{C} D^{\alpha}$ denotes Caputo fractional derivative of order $\alpha$, whose definition will be given in Definition 2.1 in the next section. As shown in [12], if $f(t, u(t))$ is continuous and satisfies the Lipschitz condition with respect to $u$, then equation (1.1) possesses a unique solution in $C[0, T]$. For this case, (1.1) combined with the initial condition is equivalent to the following Volterra-type integral equation:

$$
u(t)=u_{0}+\frac{1}{\Gamma(\alpha)} \int_{0}^{t}(t-\xi)^{\alpha-1} f(\xi, u(\xi)) \mathrm{d} \xi, \quad t \in(0, T] .
$$

With respect to numerical approximations for (1.2), two general approaches, called product integration method and fractional linear multistep methods, have been widely discussed. In these cases, a general discrete form of (1.2) is written as

$$
u_{n}=u_{0}+(\Delta t)^{\alpha} \sum_{j=0}^{n} \omega_{n-j} f\left(t_{j}, u_{j}\right)+(\Delta t)^{\alpha} \sum_{j=0}^{m} w_{n, j} f\left(t_{j}, u_{j}\right), \quad n \geq k
$$

with fixed $m \in \mathbb{N}$. Fractional linear multistep methods were originally proposed in [30] in the mid eighties of the last century. This type of methods construct the convolution quadrature weights $\left\{\omega_{j}\right\}_{j=0}^{\infty}$ satisfying

$$
\sum_{j=0}^{\infty} \omega_{j} \xi^{j}=\left(\frac{\sigma(1 / \xi)}{\rho(1 / \xi)}\right)^{\alpha}
$$

where $(\rho, \sigma)$ denote the classical implicit linear multistep formulae. For the motivation behind this idea we refer to [28]. [29] and [31] discuss the accuracy and stability properties of this type of methods. We can see they highly benefit from those of the corresponding multistep methods. Another more straightforward approach to generate the weights $\left\{\omega_{j}\right\}$ and $\left\{w_{n, j}\right\}$ is based on product integration, that is to replace the integrand $f(\xi, u(\xi))$ by some piecewise interpolation polynomials, and construct their fractional integrals of order $\alpha$ as approximations of the integral in (1.2). On the accuracy and efficiency of these methods applied to some Volterra-type integral equations with irregular kernels, we can refer to [9, $11,13,26]$ and [6,24]. In addition, [18] applies exponential integrators to fractional order problems. Generalized Adams methods and so-called $m$-steps methods are utilized by [1,2].

Under the framework of product integration, recently, some new numerical approximations of the Caputo fractional derivative of order $\alpha \in(0,1)$, named $L 1$ method [25], L1-2 method [17], $L 2-1_{\sigma}$ method [4] and method [32], were proposed and applied for solving timefractional differential equations. These methods are based on piecewise linear or quadratic interpolating polynomials approximations. In this paper, we generalize the approach by improving the degree of the piecewise polynomial to $3 \leq k \leq 6$ to approximate the function that possesses suitable smoothness. For this situation higher order of accuracy can be obtained. We establish local truncation errors and global errors estimates of the numerical schemes for (1.1) in detail. In addition, we mainly study the numerical stability of the $L 1$ method, L1-2 method, method in [32] and higher-order methods proposed in this paper. We 
apply the technique in [31] to the investigation of the stability regions of this type of numerical methods. Further, we give rigorous proof that $L 1$ method, L1-2 method and method in [32] possess $A\left(\frac{\pi}{2}\right)$-stability. Numerical experiments confirm our theoretical analyses and show that this class of methods are $A(\theta)$-stable uniformly for $0<\alpha<1$, and for some specific $\alpha$, $A$-stability can even be obtained.

The paper is organized as follows. Section 2 introduces numerical approximations of the Caputo fractional derivative of order $\alpha \in(0,1)$, and applies them to the discretization of problem (1.1). The local truncation errors of the proposed methods are discussed. Sections 3 and 4 respectively treat the stability and convergence of the discrete methods. In Sect. 5, numerical experiments confirm our theoretical considerations with respect to order of convergence and stability restrictions.

\section{Approximations of Caputo Fractional Derivatives Using Continuous Piecewise Polynomials}

We first introduce fractional derivatives in the Caputo sense:

Definition 2.1 ([12]) Let $\alpha>0$, and $n=\lceil\alpha\rceil$, the $\alpha$-th order Caputo fractional derivative of a function $u(t)$ on $[0, T]$ is defined by

$$
{ }^{C} D^{\alpha} u(t)=\frac{1}{\Gamma(n-\alpha)} \int_{0}^{t} \frac{u^{(n)}(\xi)}{(t-\xi)^{\alpha-n+1}} \mathrm{~d} \xi
$$

whenever $u^{(n)}(t) \in L^{1}[0, T]$. In particular, the Caputo fractional derivative of order $\alpha \in$ $(0,1)$ is defined by

$$
{ }^{C} D^{\alpha} u(t)=\frac{1}{\Gamma(1-\alpha)} \int_{0}^{t}(t-\xi)^{-\alpha} u^{(1)}(\xi) \mathrm{d} \xi
$$

whenever $u^{(1)}(t) \in L^{1}[0, T]$.

Next, we will derive a class of numerical approximations of the Caputo fractional derivative of order $\alpha \in(0,1)$ by constructing a series of continuous piecewise polynomials. The main idea is as follows.

Let $\mathscr{I}=[0, T]$ be an interval and the $M+1$ nodes $\left\{t_{i}\right\}_{i=0}^{M}$ define a partition

$$
0=t_{0}<t_{1} \cdots<t_{M-1}<t_{M}=T .
$$

Assume that $p_{j, q}^{k}(t)$ are a class of polynomials of degree $k \geq 1$ with compact supports $\mathscr{I}_{j}=\left[t_{j-1}, t_{j}\right]$. Their coefficients are uniquely determined by the following $k+1$ conditions

$$
p_{j, q}^{k}\left(t_{n}\right)=u\left(t_{n}\right), \quad n=j+q-1, j+q-2, \ldots, j+q-k-1 .
$$

Here the index $q$ records the number of shifts of the $k+1$ interpolating nodes $\left\{t_{n}\right\}_{n=j-1-k}^{j-1}$, and the sign of $q$ indicates the direction of the shift. Then we have

$$
p_{j, q}^{k}(t)=\sum_{n=j+q-k-1}^{j+q-1} u\left(t_{n}\right) \prod_{\substack{m=j+q-k-1 \\ m \neq n}}^{j+q-1} \frac{t-t_{m}}{t_{n}-t_{m}}, \quad t \in \mathscr{I}_{j} .
$$


If the partition (2.3) is equidistant, i.e., $t_{n}=n \Delta t, 0 \leq n \leq M$ and $\Delta t=\frac{T}{M}$, then (2.5) can be written as

$$
p_{j, q}^{k}(t)=\sum_{n=0}^{k} \frac{\nabla^{n} u\left(t_{j+q-1}\right)}{n !(\Delta t)^{n}} \prod_{l=0}^{n-1}\left(t-t_{j+q-1-l}\right), \quad t \in \mathscr{I}_{j} .
$$

For convenience of notation, let $t=t_{j-1}+s \Delta t$, we get

$$
p_{j, q}^{k}(t)=\sum_{r=0}^{k}\left(\begin{array}{c}
s-q+r-1 \\
r
\end{array}\right) \nabla^{r} u\left(t_{j+q-1}\right), \quad 0<s<1,
$$

where $\left(\begin{array}{c}s-q+r-1 \\ r\end{array}\right)$ denotes a binomial coefficient, and the $r$-th order backward difference operators $\nabla^{r}$ satisfy

$$
\nabla^{0} u\left(t_{i}\right)=u\left(t_{i}\right), \quad \nabla^{r} u\left(t_{i}\right)=\nabla^{r-1} u\left(t_{i}\right)-\nabla^{r-1} u\left(t_{i-1}\right), \quad r \geq 1 .
$$

Let

$$
C_{p}^{k}(\mathscr{I})=\left\{v(t) \in C(\mathscr{I}): \quad v(t)=\sum_{l=0}^{k} a_{j, l} t^{l} \text { on } \mathscr{I}_{j}\right\}
$$

be the space of continuous piecewise polynomials of degree at most $k$. On the uniform grid, we construct a class of polynomials of the form

$$
P_{i}^{k}(t)=\sum_{j=1}^{k-i} p_{j, k-j}^{k-1}(t)+\sum_{j=k}^{n} p_{j-i+1, i}^{k}(t)+\sum_{j=n-i+2}^{n} p_{j, n+1-j}^{k}(t),
$$

where $1 \leq i \leq k \leq 6$ and $t \in\left(t_{n-1}, t_{n}\right]$ for $1 \leq n \leq M \cdot \sum_{j=1}^{k-i} p_{j, k-j}^{k-1}(t)=0$ and $\sum_{j=n-i+2}^{n} p_{j, n+1-j}^{k}(t)=0$ if $k-i<1$ and $n-i+2>n$, respectively. Then $P_{i}^{k}(t)$ are considered as approximations of the function $u(t)$ in (2.2) in the space $C_{p}^{k}(\mathscr{I})$.

Correspondingly, we propose the operator

$$
D_{k, i}^{\alpha} u(t)=\frac{1}{\Gamma(1-\alpha)} \int_{0}^{t}(t-\xi)^{-\alpha} \frac{\mathrm{d} P_{i}^{k}}{\mathrm{~d} \xi} \mathrm{d} \xi
$$

for $t \in \mathscr{I}$ as an approximation to (2.2). If $t=t_{n}$, we rewritten (2.9) as

$$
\begin{aligned}
D_{k, i}^{\alpha} u_{n} & =\frac{(\Delta t)^{-\alpha}}{\Gamma(1-\alpha)} \sum_{j=1}^{n} \int_{0}^{1}(n-j+1-s)^{-\alpha} \frac{\mathrm{d} P_{i}^{k}\left(t_{j-1}+s \Delta t\right)}{\mathrm{d} s} \mathrm{~d} s \\
& =(\Delta t)^{-\alpha} \sum_{j=0}^{k-1} w_{n, j}^{(k, i)} u_{j}+(\Delta t)^{-\alpha} \sum_{j=0}^{n} \omega_{n-j}^{(k, i)} u_{j},
\end{aligned}
$$

where $u_{n}:=u\left(t_{n}\right)$.

Remark 1 The construction of $P_{i}^{k}(t)$ in (2.8) mainly depends on the continuity requirement on the interval $\mathscr{I}$, i.e., the interpolation conditions

$$
p_{j, q}^{k}\left(t_{n}\right)=u\left(t_{n}\right), \quad n=j-1, j
$$


should be satisfied. This means that on each $\mathscr{I}_{j}$, the conditions $j+q-1 \geq j$ and $j+q-k-1 \leq$ $j-1$ in (2.4) should be satisfied, which yields $1 \leq q \leq k$. Therefore, for $k=1$, there is only one piecewise polynomial, denoted by $P_{1}^{1}(t)$, in the space $C_{p}^{1}(\mathscr{I})$. Moreover, (2.8) yields

$$
P_{1}^{1}(t)=\sum_{j=1}^{n} p_{j, 1}^{1}(t)
$$

It is easy to see that $P_{1}^{1}(t)$ coincides with the known $L 1$ method proposed in [25]. For $k=2$, we can choose $p_{j, 1}^{1}(t), p_{j, 1}^{2}(t)$ and $p_{j, 2}^{2}(t)$ on each $\mathscr{I}_{j}$ such that (2.11) holds. To preserve the convolution property as much as possible, here we provide two cases

$$
P_{1}^{2}(t)=p_{1,1}^{1}(t)+\sum_{j=2}^{n} p_{j, 1}^{2}(t) \quad \text { and } \quad P_{2}^{2}(t)=\sum_{j=1}^{n-1} p_{j, 2}^{2}(t)+p_{n, 1}^{2}(t)
$$

where $t \in\left(t_{n-1}, t_{n}\right]$. The two cases in (2.12) coincide with the approximate methods discussed in [17] and [32], respectively. In addition, as presented in (2.8), we restrict our further discussion to the case $i \leq k$. Because under the condition, the corresponding discrete operators $D_{k, i}^{\alpha} u_{n}$ in (2.10) can be computed with the least starting values.

In the following part, we present the explicit representations of the weight coefficients $\left\{w_{n, j}^{(k, i)}\right\}$ and $\left\{\omega_{j}^{(k, i)}\right\}$ for $1 \leq i \leq k \leq 3$ as examples. Note that in the case $1 \leq i \leq k \leq 2$, the weight coefficients have been derived by $[17,25,32]$ in a similar way. Here we rewrite them into the form of integrals for convenience of further theoretical analyses. First, we define a class of integrals of the form

$$
I_{n, q}^{r}= \begin{cases}\frac{1}{\Gamma(1-\alpha)} \int_{0}^{1}(n+1-s)^{-\alpha} \mathrm{d}\left(\begin{array}{c}
s-q+r-1 \\
r
\end{array}\right), & n \geq 0 \\
0, & n<0\end{cases}
$$

where $q, r \in \mathbb{N}^{+}$and $n \in \mathbb{Z}$. If we denote

$$
\begin{aligned}
I_{n} & :=I_{n, q}^{1}, \quad \forall q=1,2, \ldots, \\
\nabla^{k} I_{n, q}^{r} & =\nabla^{k-1} I_{n, q}^{r}-\nabla^{k-1} I_{n-1, q}^{r}, \quad \forall k \in \mathbb{N}^{+},
\end{aligned}
$$

then the weight coefficients can be expressed as

$$
\left\{\begin{aligned}
(k, i)=(1,1): & w_{m, 0}=-I_{m}, \quad m \geq 1, \quad \omega_{n}=\nabla I_{n}, \quad n \geq 0, \\
(k, i)=(2,1): & w_{m, 0}=2 I_{m-1,1}^{2}-I_{m, 1}^{2}-I_{m}, \quad w_{m, 1}=-I_{m-1,1}^{2}, \quad m \geq 2, \\
& \omega_{n}=\nabla I_{n}+\nabla^{2} I_{n, 1}^{2}, \quad n \geq 0, \\
(k, i)=(2,2): & w_{m, 0}=-\nabla I_{m+1,1}^{2}+I_{m, 2}^{2}, \quad w_{m, 1}=-I_{m, 1}^{2}, \quad m \geq 2, \\
& \omega_{0}=I_{0}+I_{1}+I_{0,1}^{2}+I_{1,2}^{2}, \quad \omega_{1}=\nabla I_{2}-I_{0}+I_{2,2}^{2}-2 I_{0,1}^{2}-2 I_{1,2}^{2}, \\
& \omega_{2}=\nabla I_{3}+\nabla^{2} I_{3,2}^{2}+I_{0,1}^{2}, \quad \omega_{n}=\nabla I_{n+1}+\nabla^{2} I_{n+1,2}^{2}, \quad n \geq 3,
\end{aligned}\right.
$$

and 


$$
\begin{aligned}
(k, i)= & (3,1): \\
& \left\{\begin{array}{l}
w_{m, 0}=-\nabla I_{m}-I_{m, 1}^{2}+2 I_{m-1,1}^{2}+I_{m-1,2}^{2}-I_{m, 1}^{3}+3 I_{m-1,1}^{3}-3 I_{m-2,1}^{3}, \\
w_{m, 1}=-2 I_{m-1}-2 I_{m-1,2}^{2}-I_{m-1,1}^{2}-I_{m-1,1}^{3}+3 I_{m-2,1}^{3}, \\
w_{m, 2}=I_{m-1}+I_{m-1,2}^{2}-I_{m-2,1}^{3}, \quad m \geq 3, \\
\omega_{n}=\nabla I_{n}+\nabla^{2} I_{n, 1}^{2}+\nabla^{3} I_{n, 1}^{3}, \quad n \geq 0,
\end{array}\right.
\end{aligned}
$$

$(k, i)=(3,2):$

$$
\left\{\begin{array}{l}
w_{m, 0}=-\nabla I_{m+1}-I_{m+1,2}^{2}+2 I_{m, 2}^{2}-I_{m+1,2}^{3}+3 I_{m, 2}^{3}-3 I_{m-1,2}^{3}, \\
w_{m, 1}=-I_{m}-I_{m, 2}^{2}-I_{m, 2}^{3}+3 I_{m-1,2}^{3} \\
w_{m, 2}=-I_{m-1,2}^{3}, \quad m \geq 3 \\
\omega_{0}=I_{0}+I_{1}+I_{1,2}^{2}+I_{0,1}^{2}+I_{1,2}^{3}+I_{0,1}^{3} \\
\omega_{1}=\nabla I_{2}-I_{0}+I_{2,2}^{2}-2 I_{1,2}^{2}-2 I_{0,1}^{2}+I_{2,2}^{3}-3 I_{1,2}^{3}-3 I_{0,1}^{3} \\
\omega_{2}=\nabla I_{3}+\nabla^{2} I_{3,2}^{2}+I_{0,1}^{2}+I_{3,2}^{3}-3 I_{2,2}^{3}+3 I_{1,2}^{3}+3 I_{0,1}^{3} \\
\omega_{3}=\nabla I_{4}+\nabla^{2} I_{4,2}^{2}+\nabla^{3} I_{4,2}^{3}-I_{0,1}^{3}, \\
\omega_{n}=\nabla I_{n+1}+\nabla^{2} I_{n+1,2}^{2}+\nabla^{3} I_{n+1,2}^{3}, \quad n \geq 4
\end{array}\right.
$$

$(k, i)=(3,3)$ :

$$
\left\{\begin{array}{l}
w_{m, 0}=-\nabla I_{m+2}-\nabla^{2} I_{m+2,3}^{2}-I_{m+2,3}^{3}+3 I_{m+1,3}^{3}-3 I_{m, 3}^{3}, \\
w_{m, 1}=-\nabla I_{m+1}-I_{m+1,3}^{2}+2 I_{m, 3}^{2}-I_{m+1,3}^{3}+3 I_{m, 3}^{3}, \\
w_{m, 2}=-I_{m}-I_{m, 3}^{2}-I_{m, 3}^{3}, \quad m \geq 3, \\
\omega_{0}=I_{0}+I_{1}+I_{2}+I_{0,1}^{2}+I_{1,2}^{2}+I_{2,3}^{2}+I_{0,1}^{3}+I_{1,2}^{3}+I_{2,3}^{3}, \\
\omega_{1}=\nabla I_{3}-I_{0}-I_{1}+I_{3,3}^{2}-2 I_{2,3}^{2}-2 I_{1,2}^{2}-2 I_{0,1}^{2}+I_{3,3}^{3}-3 I_{2,3}^{3}-3 I_{1,2}^{3}-3 I_{0,1}^{3}, \\
\omega_{2}=\nabla I_{4}+\nabla^{2} I_{4,3}^{2}+I_{1,2}^{2}+I_{0,1}^{2}+I_{4,3}^{3}-3 I_{3,3}^{3}+3 I_{2,3}^{3}+3 I_{0,1}^{3}+3 I_{1,2}^{3}, \\
\omega_{3}=\nabla I_{5}+\nabla^{2} I_{5,3}^{2}+\nabla^{3} I_{5,3}^{3}-I_{1,2}^{3}-I_{0,1}^{3}, \\
\omega_{n}=\nabla I_{n+2}+\nabla^{2} I_{n+2,3}^{2}+\nabla^{3} I_{n+2,3}^{3}, \quad n \geq 4 .
\end{array}\right.
$$

It can be observed that when $\alpha \rightarrow 1$, the operator $D_{k, i}^{\alpha} u_{n}$ in (2.10) recovers the $k$-step BDF method.

Using (2.10), we construct the discrete schemes

$$
D_{k, i}^{\alpha} u_{n}=f\left(t_{n}, u_{n}\right), \quad n \geq k,
$$

as approximations of Eq. (1.1). If starting values are given, then we define the local truncation errors of the $n$-th step by

$$
\tau_{n}^{(k, i)}=D_{k, i}^{\alpha} u\left(t_{n}\right)-{ }^{C} D^{\alpha} u\left(t_{n}\right), \quad n \geq k,
$$

where $u(t)$ is the exact solution of (1.1).

Theorem 2.1 Let $0<\alpha<1$ and $1 \leq k \leq 6$. If $u(t) \in C^{k+1}[0, T]$, then for $1 \leq i<k$, it holds that

$$
\tau_{n}^{(k, i)}=O\left(\left(t_{n-k+i}\right)^{-\alpha-1} \Delta t^{k+1}+\Delta t^{k+1-\alpha}\right), \quad n \geq k
$$


In particular,

$$
\tau_{n}^{(k, k)}=O\left(\Delta t^{k+1-\alpha}\right), \quad n \geq k
$$

Proof From (2.7), we have

$$
p_{j, q}^{k}(t)-u(t)=u^{(k+1)}\left(\xi_{j}\right)\left(\begin{array}{c}
s-q+k \\
k+1
\end{array}\right)(\Delta t)^{k+1}
$$

for $t=t_{j-1}+s \Delta t, 0 \leq s \leq 1$, where $t_{j+q-k-1} \leq \xi_{j} \leq t_{j+q-1}$.

Inspired by [17], integration by part yields

$$
\begin{aligned}
\tau_{n}^{(k, i)} & =\frac{1}{\Gamma(1-\alpha)} \sum_{j=1}^{n} \int_{t_{j-1}}^{t_{j}}\left(t_{n}-t\right)^{-\alpha}\left(\frac{\mathrm{d} P_{i}^{k}(t)}{\mathrm{d} t}-\frac{\mathrm{d} u(t)}{\mathrm{d} t}\right) \mathrm{d} t \\
& =\frac{-\alpha}{\Gamma(1-\alpha)} \sum_{j=1}^{n} \int_{t_{j-1}}^{t_{j}}\left(t_{n}-t\right)^{-\alpha-1}\left(P_{i}^{k}(t)-u(t)\right) \mathrm{d} t \\
& =\frac{-\alpha(\Delta t)^{-\alpha}}{\Gamma(1-\alpha)} \sum_{j=1}^{n} \int_{0}^{1}(n-j+1-s)^{-\alpha-1}\left(P_{i}^{k}\left(t_{j-1}+s \Delta t\right)-u\left(t_{j-1}+s \Delta t\right)\right) \mathrm{d} s
\end{aligned}
$$

for $n \geq k$. Substituting (2.8) and (2.21) into the last formula of (2.22) and taking $i=k$, we get

$$
\begin{aligned}
\left|\tau_{n}^{(k, k)}\right| \leq & \frac{\alpha(\Delta t)^{k+1-\alpha}}{\Gamma(1-\alpha)} \max _{\xi \in \mathscr{I}}\left|u^{(k+1)}(\xi)\right|\left(\sum_{j=1}^{n-k+1}\left|\int_{0}^{1}(n-j+1-s)^{-\alpha-1}\left(\begin{array}{c}
s \\
k+1
\end{array}\right) \mathrm{d} s\right|\right. \\
& \left.+\sum_{j=n-k+2}^{n}\left|\int_{0}^{1}(n-j+1-s)^{-\alpha-1}\left(\begin{array}{c}
s+k-n-1+j \\
k+1
\end{array}\right) \mathrm{d} s\right|\right),
\end{aligned}
$$

and for $1 \leq i \leq k-1$,

$$
\begin{aligned}
& \left|\tau_{n}^{(k, i)}\right| \leq \frac{\alpha(\Delta t)^{-\alpha}}{\Gamma(1-\alpha)}\left((\Delta t)^{k} \max _{\xi \in \cup_{j=1}^{k-i} \mathscr{I}_{j}}\left|u^{(k)}(\xi)\right| \sum_{j=1}^{k-i}\left|\int_{0}^{1}(n-j+1-s)^{-\alpha-1}\left(\begin{array}{c}
s+j-1 \\
k
\end{array}\right) \mathrm{d} s\right|\right. \\
& +(\Delta t)^{k+1} \max _{\xi \in \mathscr{I}}\left|u^{(k+1)}(\xi)\right| \sum_{j=k-i+1}^{n-i+1}\left|\int_{0}^{1}(n-j+1-s)^{-\alpha-1}\left(\begin{array}{c}
s+k-i \\
k+1
\end{array}\right) \mathrm{d} s\right| \\
& \left.+(\Delta t)^{k+1} \max _{\xi \in \mathscr{I}}\left|u^{(k+1)}(\xi)\right| \sum_{j=n-i+2}^{n}\left|\int_{0}^{1}(n-j+1-s)^{-\alpha-1}\left(\begin{array}{c}
s+k-n-i+j \\
k+1
\end{array}\right) \mathrm{d} s\right|\right) .
\end{aligned}
$$

Since for any $q \leq k$ with $q, k \in \mathbb{N}^{+}$, the factor $(1-s)$ is included in $\left(\begin{array}{c}s-q+k \\ k+1\end{array}\right)$ and the term $\frac{1}{1-s}\left(\begin{array}{c}s-q+k \\ k+1\end{array}\right)$ is bounded for $0 \leq s \leq 1$, we have 


$$
\begin{aligned}
\left|\tau_{n}^{(k, k)}\right| & \leq \frac{\alpha(\Delta t)^{k+1-\alpha}}{\Gamma(1-\alpha)} C^{(k)} \sum_{j=1}^{n} \int_{0}^{1}(n-j+1-s)^{-\alpha-1}(1-s) \mathrm{d} s \\
& \leq \frac{\alpha(\Delta t)^{k+1-\alpha}}{\Gamma(1-\alpha)} C^{(k)}\left(\sum_{j=1}^{n-1} \int_{0}^{1}(n-j+1-s)^{-\alpha-1} \mathrm{~d} s+\int_{0}^{1}(1-s)^{-\alpha} \mathrm{d} s\right) \\
& \leq(\Delta t)^{k+1-\alpha} C^{(k)}\left(\frac{1}{\Gamma(1-\alpha)}+\frac{1}{\Gamma(2-\alpha)}\right)
\end{aligned}
$$

where $C^{(k)}$ are bounded and depend on $u^{(k+1)}$ and $k$. On the other hand, if $i<k$, it holds that

$$
\begin{aligned}
\left|\tau_{n}^{(k, i)}\right| \leq & \frac{\alpha}{\Gamma(1-\alpha)} C^{(k, i)}\left((\Delta t)^{k-\alpha} \sum_{j=1}^{k-i} \int_{0}^{1}(n-j+1-s)^{-\alpha-1} \mathrm{~d} s\right. \\
& \left.+(\Delta t)^{k+1-\alpha} \sum_{j=1}^{n} \int_{0}^{1}(n-j+1-s)^{-\alpha-1}(1-s) \mathrm{d} s\right) \\
\leq & C^{(k, i)}\left(\frac{\alpha}{\Gamma(1-\alpha)}(\Delta t)^{k+1}(k-i)\left(t_{n-k+i}\right)^{-\alpha-1}\right. \\
& \left.+(\Delta t)^{k+1-\alpha}\left(\frac{1}{\Gamma(1-\alpha)}+\frac{1}{\Gamma(2-\alpha)}\right)\right),
\end{aligned}
$$

where $C^{(k, i)}$ are constants dependent on $u^{(k)}, u^{(k+1)}$ and $k, i$.

\section{Stability Analysis}

To analyse the stability of discrete schemes (2.17) with initial value $u(0)=u_{0}$, we apply (2.9) to the test equation

$$
{ }^{C} D^{\alpha} u(t)=\lambda u(t), \quad \lambda \in \mathbb{C}
$$

and obtain

$$
D_{k, i}^{\alpha} u_{n}=\lambda u_{n}, \quad n \geq k .
$$

We rewrite (3.2) as the formal power series form

$$
\sum_{n=0}^{\infty} D_{k, i}^{\alpha} u_{n+k} \xi^{n}=\lambda \sum_{n=0}^{\infty} u_{n+k} \xi^{n} .
$$

Replaced by (2.10), formula (3.3) becomes

$$
\omega^{(k, i)}(\xi) \mathscr{U}(\xi)=z \mathscr{U}(\xi)+g^{(k, i)}(\xi),
$$

where $z:=\lambda(\Delta t)^{\alpha}$. The above notations are defined as

$$
\begin{gathered}
\mathscr{U}(\xi)=\sum_{n=0}^{\infty} u_{n+k} \xi^{n}, \quad \omega^{(k, i)}(\xi)=\sum_{n=0}^{\infty} \omega_{n}^{(k, i)} \xi^{n}, \\
g^{(k, i)}(\xi)=-\sum_{j=0}^{k-1} u_{j} \sum_{n=0}^{\infty}\left(w_{n+k, j}^{(k, i)}+\omega_{n+k-j}^{(k, i)}\right) \xi^{n} .
\end{gathered}
$$


Theorem 3.1 The stability region of (3.2) with $1 \leq i \leq k \leq 6$ is $\mathbb{C} \backslash\left\{\omega^{(k, i)}(\xi):|\xi| \leq 1\right\}$.

Remark 2 The stability region of a method applied to the test equation (3.1) is the set of $z=\lambda(\Delta t)^{\alpha} \in \mathbb{C}$ with $\Delta t>0$ such that $u_{n} \rightarrow 0$ as $n \rightarrow \infty$ whenever the starting values $u_{0}, \ldots, u_{k-1}$ are bounded.

Proof If we denote the stability region of (3.2) by $S^{(k, i)}$, then the proof of $S^{(k, i)}=$ $\mathbb{C} \backslash\left\{\omega^{(k, i)}(\xi):|\xi| \leq 1\right\}$ is equivalent to proving $S^{(k, i)} \supseteq \mathbb{C} \backslash\left\{\omega^{(k, i)}(\xi):|\xi| \leq 1\right\}$ and $S^{(k, i)} \subseteq \mathbb{C} \backslash\left\{\omega^{(k, i)}(\xi):|\xi| \leq 1\right\}$, i.e., to prove that if $z \in \mathbb{C} \backslash\left\{\omega^{(k, i)}(\xi):|\xi| \leq 1\right\}$, then $z \in S^{(k, i)}$ and if $z \notin \mathbb{C} \backslash\left\{\omega^{(k, i)}(\xi):|\xi| \leq 1\right\}$, then $z \notin S^{(k, i)}$.

On the one hand, if $z \in \mathbb{C} \backslash\left\{\omega^{(k, i)}(\xi):|\xi| \leq 1\right\}$ and $|z| \leq 1$, then $z-\omega^{(k, i)}(\xi) \neq 0$ for $|\xi| \leq 1$. Thus, by Lemmas A.4, A.5 and Theorem A.1, the coefficient sequence of the reciprocal of $z-\omega^{(k, i)}(\xi)$ is in $l^{1}$ and the coefficient sequence of $g^{(k, i)}(\xi)$ tends to zero.

If $|z|>1$, formula (3.4) can be rewritten as

$$
\mathscr{U}(\xi)=\frac{\frac{g^{(k, i)}(\xi)}{z}}{\frac{\omega^{(k, i)}(\xi)}{z}-1},
$$

in which case the coefficient sequence of the reciprocal of $\frac{\omega^{(k, i)}(\xi)}{z}-1$ is in $l^{1}$, and the coefficient sequence of $\frac{g^{(k, i)}(\xi)}{z}$ converges to zero. In addition, if $\lim _{n \rightarrow \infty} \sum_{j=0}^{n}\left|l_{i}\right|=L<+\infty$ and $\lim _{j \rightarrow \infty} c_{j}=0$, then $\lim _{n \rightarrow \infty} \sum_{j=0}^{n} l_{n-j} c_{j}=0$ follows. This implies $u_{n} \rightarrow 0$ as $n \rightarrow \infty$.

On the other hand, assume that $z=\omega^{(k, i)}\left(\xi_{0}\right)$ for some $\left|\xi_{0}\right| \leq 1$, then formula (3.4) becomes

$$
\left(\omega^{(k, i)}(\xi)-\omega^{(k, i)}\left(\xi_{0}\right)\right) \mathscr{U}(\xi)=g^{(k, i)}(\xi) .
$$

If applying the methods (2.10) on a constant function, we obtain from Theorem 2.1 that the corresponding truncation errors are zero, which leads to

$$
\sum_{j=0}^{k-1} w_{n, j}^{(k, i)}+\sum_{j=0}^{n} \omega_{n-j}^{(k, i)}=0, \quad n \geq k,
$$

and consequently,

$$
\begin{aligned}
& \sum_{n=k}^{\infty}\left(\sum_{j=0}^{k-1} w_{n, j}^{(k, i)}+\sum_{j=0}^{n} \omega_{n-j}^{(k, i)}\right) \xi^{n-k} \\
& =\sum_{n=0}^{\infty}\left(\sum_{j=0}^{k-1} w_{n+k, j}^{(k, i)}+\sum_{j=0}^{n+k} \omega_{n+k-j}^{(k, i)}\right) \xi^{n} \\
& =\sum_{n=0}^{\infty} \sum_{j=0}^{k-1}\left(w_{n+k, j}^{(k, i)}+\omega_{n+k-j}^{(k, i)}\right) \xi^{n}+\frac{\omega^{(k, i)}(\xi)}{1-\xi}=0 .
\end{aligned}
$$

Assume that $u_{0}=\cdots=u_{k-1} \neq 0$, with the expression of $g^{(k, i)}(\xi)$, we find $g^{(k, i)}(\xi)=$ $u_{0} \frac{\omega^{(k, i)}(\xi)}{1-\xi}$. If $\omega^{(k, i)}\left(\xi_{0}\right)=0$, then $\mathscr{U}(\xi)=\frac{u_{0}}{1-\xi}$, which means that $u_{n}=u_{0}$ for all $n \in \mathbb{N}$. If $\omega^{(k, i)}\left(\xi_{0}\right) \neq 0$, then we have 


$$
\mathscr{U}(\xi)(1-\xi) \frac{\omega^{(k, i)}(\xi)-\omega^{(k, i)}\left(\xi_{0}\right)}{\xi-\xi_{0}}=u_{0} \frac{\omega^{(k, i)}(\xi)-\omega^{(k, i)}\left(\xi_{0}\right)}{\xi-\xi_{0}}+u_{0} \frac{\omega^{(k, i)}\left(\xi_{0}\right)}{\xi-\xi_{0}} .
$$

If assume that $u_{n} \rightarrow 0$ as $n \rightarrow \infty$, then from Lemma A.6, it follows that the coefficient sequence of $(1-\xi) \frac{\omega^{(k, i)}(\xi)-\omega^{(k, i)}\left(\xi_{0}\right)}{\xi-\xi_{0}}$ is in the space $l^{1}$. This indicates that the coefficient sequence of $\mathscr{U}(\xi)(1-\xi) \frac{\omega^{(1,1)}(\xi)-\omega^{(1,1)}\left(\xi_{0}\right)}{\xi-\xi_{0}}$ tends to zero. In addition, Lemma A.3 presents that the coefficient sequence of $\frac{\omega^{(k, i)}(\xi)-\omega^{(k, i)}\left(\xi_{0}\right)}{\xi-\xi_{0}}$ converges to zero. However, the divergence of the coefficient sequence of $\frac{1}{\xi-\xi_{0}}$ for $\left|\xi_{0}\right| \leq 1$ leads to a contradiction. Thus, there exist some nonzero bounded initial values $\left\{u_{i}\right\}_{i=0}^{k-1}$ such that $u_{n} \nrightarrow \rightarrow 0$ as $n \rightarrow \infty$, which indicates $z \notin \mathrm{S}^{(k, i)}$.

Analogous to the $A(\theta)$-stability for classical ODE mentioned in [19], we define $A(\theta)$ stability of methods for fractional ODE.

Definition 3.1 A method is said to be $A(\theta)$-stable with $\theta \in\left[0, \pi-\frac{\alpha \pi}{2}\right)$ and $0<\alpha<1$, if the sector

$$
S_{\theta}=\{z:|\arg (-z)| \leq \theta, \quad z \neq 0\}
$$

is contained in the stability region.

Theorem 3.2 The method (3.2) is A( $\left.\frac{\pi}{2}\right)$-stable for $1 \leq i \leq k \leq 2$.

Proof For $\theta=\frac{\pi}{2}$ in Definition 3.1, it suffices to prove $\mathrm{S}_{\frac{\pi}{2}} \subseteq S^{(k, i)}$ for $1 \leq i \leq k \leq 2$, namely, to prove $\omega^{(k, i)}(\xi)=0$ for some $|\xi| \leq 1$ and $\operatorname{Re}\left(\omega^{(k, i)}(\xi)\right)>0$ otherwise.

First of all, it can be readily verified that $\omega^{(k, i)}(1)=0$, which implies $0 \notin \mathrm{S}_{\frac{\pi}{2}}$. Next we prove the results for the case $(k, i)=(1,1),(k, i)=(2,1)$ and $(k, i)=(2,2)$ separately.

Case $(k, i)=(1,1)$ : from the expression of $\omega^{(1,1)}(\xi)$, we obtain

$$
\omega^{(1,1)}(\xi)=I_{0}+\sum_{j=1}^{\infty} \nabla I_{j} \xi^{j}=(1-\xi) I(\xi)
$$

where $I(\xi)=\sum_{n=0}^{\infty} I_{n} \xi^{n}$. Lemma A.1 and Theorem A.2 yield

$$
I_{n}=\int_{0}^{1} r^{n} \mathrm{~d} \sigma(r), \quad n \in \mathbb{N},
$$

where $\sigma(r)$ is a non-decreasing function. Suppose that $|\xi|<1$, substituting (3.8) into (3.7) yields

$$
\operatorname{Re}\left(\omega^{(1,1)}(\xi)\right)=\operatorname{Re}\left((1-\xi) \sum_{n=0}^{\infty} \int_{0}^{1} r^{n} \xi^{n} \mathrm{~d} \sigma(r)\right)=\int_{0}^{1} \operatorname{Re}\left(\frac{1-\xi}{1-r \xi}\right) \mathrm{d} \sigma(r) .
$$

Let $\xi=|\xi|(\cos \theta+i \sin \theta)$, then

$$
\frac{1-\xi}{1-r \xi}=\frac{\left(1-(r+1)|\xi| \cos \theta+r|\xi|^{2}\right)+i((r-1)|\xi| \sin \theta)}{(1-r|\xi| \cos \theta)^{2}+(r|\xi| \sin \theta)^{2}} .
$$

For $0 \leq r \leq 1$ and $|\xi|<1$, we find

$$
\begin{aligned}
1-(r+1)|\xi| \cos \theta+r|\xi|^{2} & \geq \min \left((1-|\xi| \cos \theta)^{2}, 1-|\xi| \cos \theta\right), \\
1-2 r|\xi| \cos \theta+r^{2}|\xi|^{2} & \leq(1+r|\xi|)^{2} \leq 4
\end{aligned}
$$


which yield

$$
\int_{0}^{1} \operatorname{Re}\left(\frac{1-\xi}{1-r \xi}\right) d \sigma(r) \geq \frac{\min \left((1-|\xi| \cos \theta)^{2}, 1-|\xi| \cos \theta\right)}{4} I_{0} .
$$

Case $(k, i)=(2,1)$ : using the definition of $\omega^{(2,1)}(\xi)$, we observe

$$
\begin{aligned}
\omega^{(2,1)}(\xi) & =\sum_{n=0}^{\infty}\left(\nabla I_{n}+\nabla^{2} I_{n, 1}^{2}\right) \xi^{n} \\
& =(1-\xi) I(\xi)+(1-\xi)^{2} I_{1}^{2}(\xi) \\
& =(1-\xi)\left(I(\xi)-2 I_{1}^{2}(\xi)+(3-\xi) I_{1}^{2}(\xi)\right),
\end{aligned}
$$

where

$$
I(\xi)=\sum_{n=0}^{\infty} I_{n} \xi^{n}, \quad I_{1}^{2}(\xi)=\sum_{n=0}^{\infty} I_{n, 1}^{2} \xi^{n}
$$

Lemmas A.1, A.2 and Theorem A.2 yield

$$
I_{n}-2 I_{n, 1}^{2}=\int_{0}^{1} r^{n} \mathrm{~d} v(r), \quad n=0,1, \ldots
$$

and

$$
I_{n, 1}^{2}=\int_{0}^{1} r^{n} \mathrm{~d} \gamma(r), \quad n=0,1, \ldots
$$

where both $v$ and $\gamma$ are non-decreasing functions. Then for $|\xi|<1$,

$$
\operatorname{Re}\left(\omega^{(2,1)}(\xi)\right)=\int_{0}^{1} \operatorname{Re}\left(\frac{1-\xi}{1-r \xi}\right) \mathrm{d} v(r)+\int_{0}^{1} \operatorname{Re}\left(\frac{(1-\xi)(3-\xi)}{1-r \xi}\right) \mathrm{d} \gamma(r) .
$$

Moreover,

$$
\begin{aligned}
& \frac{(1-\xi)(3-\xi)}{1-r \xi} \\
& \quad=\frac{\left(3-4|\xi| \cos \theta+|\xi|^{2} \cos 2 \theta\right)(1-r|\xi| \cos \theta)+(4-2|\xi| \cos \theta) r|\xi|^{2} \sin ^{2} \theta}{(1-r|\xi| \cos \theta)^{2}+(r|\xi| \sin \theta)^{2}} \\
& \quad+i \frac{\left(3 r-|\xi|^{2} r-4+2|\xi| \cos \theta\right)|\xi| \sin \theta}{(1-r|\xi| \cos \theta)^{2}+(r|\xi| \sin \theta)^{2}}
\end{aligned}
$$

Since

$$
3-4|\xi| \cos \theta+|\xi|^{2} \cos 2 \theta=3-4|\xi| \cos \theta+2|\xi|^{2} \cos ^{2} \theta-|\xi|^{2} \geq 2(1-|\xi| \cos \theta)^{2}
$$

we get

$$
\int_{0}^{1} \operatorname{Re}\left(\frac{(1-\xi)(3-\xi)}{1-r \xi}\right) \mathrm{d} \gamma(r) \geq \frac{\min \left((1-|\xi| \cos \theta)^{3},(1-|\xi| \cos \theta)^{2}\right)}{2} I_{0,1}^{2} .
$$


Case $(k, i)=(2,2)$ : the series $\omega^{(2,2)}(\xi)$ satisfies

$$
\begin{aligned}
\omega^{(2,2)}(\xi) & =I_{0}(1-\xi)+I_{0,1}^{2}(1-\xi)^{2}+(1-\xi) \sum_{n=0}^{\infty} I_{n+1} \xi^{n}+(1-\xi)^{2} \sum_{n=0}^{\infty} I_{n+1,2}^{2} \xi^{n} \\
& =I_{0,1}^{2}(1-\xi)(3-\xi)+\left(1-\xi^{2}\right) \sum_{n=0}^{\infty} I_{n+1,1}^{2} \xi^{n}+(1-\xi)\left(I(\xi)-2 I_{1}^{2}(\xi)\right),
\end{aligned}
$$

since for $n \geq 0$, the relation $I_{n}+I_{n, 2}^{2}=I_{n, 1}^{2}$ yields

$$
\begin{aligned}
& (1-\xi) \sum_{n=0}^{\infty} I_{n+1} \xi^{n}+(1-\xi)^{2} \sum_{n=0}^{\infty} I_{n+1,2}^{2} \xi^{n} \\
& =(1-\xi)\left(\sum_{n=0}^{\infty} I_{n+1,1}^{2} \xi^{n}-\xi \sum_{n=0}^{\infty} I_{n+1,2}^{2} \xi^{n}\right) \\
& =\left(1-\xi^{2}\right) \sum_{n=0}^{\infty} I_{n+1,1}^{2} \xi^{n}+(1-\xi) \sum_{n=0}^{\infty}\left(I_{n+1}-2 I_{n+1,1}^{2}\right) \xi^{n+1} \\
& =\left(1-\xi^{2}\right) \sum_{n=0}^{\infty} I_{n+1,1}^{2} \xi^{n}+(1-\xi)\left(I(\xi)-2 I_{1}^{2}(\xi)-\left(I_{0}-2 I_{0,1}^{2}\right)\right) .
\end{aligned}
$$

Suppose that $|\xi|<1$, substituting (3.10) and (3.11) into (3.12), we obtain

$$
\begin{aligned}
\operatorname{Re}\left(\omega^{(2,2)}(\xi)\right)= & \int_{0}^{1} \operatorname{Re}((1-\xi)(3-\xi)) \mathrm{d} \gamma(r) \\
& +\int_{0}^{1} r \operatorname{Re}\left(\frac{1-\xi^{2}}{1-r \xi}\right) \mathrm{d} \gamma(r)+\int_{0}^{1} \operatorname{Re}\left(\frac{1-\xi}{1-r \xi}\right) \mathrm{d} v(r) .
\end{aligned}
$$

Furthermore,

$$
\begin{aligned}
\frac{1-\xi^{2}}{1-r \xi}= & \frac{\left(1-|\xi|^{2} \cos 2 \theta\right)(1-r|\xi| \cos \theta)+r|\xi|^{3} \sin \theta \sin 2 \theta}{(1-r|\xi| \cos \theta)^{2}+(r|\xi| \sin \theta)^{2}} \\
& +i \frac{\left(1-|\xi|^{2} \cos 2 \theta\right) r|\xi| \sin \theta-(1-r|\xi| \cos \theta) \rho^{2} \sin 2 \theta}{(1-r|\xi| \cos \theta)^{2}+(r|\xi| \sin \theta)^{2}} .
\end{aligned}
$$

Since for $0 \leq r \leq 1$,

$$
\begin{aligned}
(1 & \left.-|\xi|^{2} \cos 2 \theta\right)(1-r|\xi| \cos \theta)+r|\xi|^{3} \sin \theta \sin 2 \theta \\
& =1-|\xi|^{2} \cos 2 \theta-r|\xi| \cos \theta+r|\xi|^{3} \cos \theta \\
& \geq\left(1-|\xi|^{2}\right)(1-|\xi||\cos \theta|),
\end{aligned}
$$

we obtain

$$
\begin{aligned}
\int_{0}^{1} r \operatorname{Re}\left(\frac{1-\xi^{2}}{1-r \xi}\right) \mathrm{d} \gamma(r) & \geq \frac{\left(1-|\xi|^{2}\right)(1-|\xi||\cos \theta|)}{4} \int_{0}^{1} r \mathrm{~d} \gamma(r) \\
& =\frac{\left(1-|\xi|^{2}\right)(1-|\xi||\cos \theta|)}{4} I_{1,1}^{2} .
\end{aligned}
$$


Finally, for $1 \leq i \leq k \leq 2$, we conclude

$$
\operatorname{Re}\left(\omega^{(k, i)}(\xi)\right) \geq \frac{\min \left((1-|\xi| \cos \theta)^{2}, 1-|\xi| \cos \theta\right)}{4} I_{0}>0, \quad|\xi|<1 .
$$

In addition, according to Lemma A.6, there exist constants $M^{(k, i)}>0$ such that

$$
\left|\omega^{(k, i)}(\xi)-\omega^{(k, i)}\left(\xi_{0}\right)\right| \leq \frac{M^{(k, i)}}{|1-\xi|}\left|\xi-\xi_{0}\right|, \quad \xi \neq 1 .
$$

This implies that $\omega^{(k, i)}(\xi)$ is continuous for $|\xi| \leq 1$ and $\xi \neq 1$. Therefore, for any fixed $\xi$ lying on the unit circle, the angle of which satisfies $\arg (\xi)=\theta_{\xi} \neq 0$, there exists a sequence $\xi_{n}=\left(1-\frac{1}{n}\right) \xi, n=1,2, \ldots$, with $\left|\xi_{n}\right|<1$, such that

$$
\operatorname{Re}\left(\omega^{(k, i)}(\xi)\right)=\lim _{n \rightarrow \infty} \operatorname{Re}\left(\omega^{(k, i)}\left(\xi_{n}\right)\right) \geq \frac{I_{0}}{4} \min \left(\left(1-\cos \theta_{\xi}\right)^{2}, 1-\cos \theta_{\xi}\right)>0 .
$$

\section{Convergence Analysis}

In this section, we will establish the error estimate for (2.17). Assume that $u(t)$ is the exact solution of (1.1), then it satisfies

$$
D_{k, i}^{\alpha} u\left(t_{n}\right)=f\left(t_{n}, u\left(t_{n}\right)\right)+\tau_{n}^{(k, i)}, \quad k \leq n \leq N,
$$

where the difference operator $D_{k, i}^{\alpha}$ and the local truncation error $\tau_{n}^{(k, i)}$ are defined by (2.10) and (2.18), respectively. Suppose that $u_{n}^{(k, i)}$ is the solution of (2.17) for each $k, i$, we denote global errors by

$$
e_{n}^{(k, i)}=u\left(t_{n}\right)-u_{n}^{(k, i)}, \quad 0 \leq n \leq N .
$$

Subtracting (2.17) by (4.1) yields

$$
D_{k, i}^{\alpha} e_{n}^{(k, i)}=\delta f_{n}^{(k, i)}+\tau_{n}^{(k, i)}, \quad k \leq n \leq N,
$$

where $\delta f_{n}^{(k, i)}=f\left(t_{n}, u\left(t_{n}\right)\right)-f\left(t_{n}, u_{n}^{(k, i)}\right)$. From (2.10) and (4.3), we have

$$
\sum_{m=0}^{k-1} w_{n, m}^{(k, i)} e_{m}^{(k, i)}+\sum_{j=0}^{n} \omega_{n-j}^{(k, i)} e_{j}^{(k, i)}=(\Delta t)^{\alpha} \delta f_{n}^{(k, i)}+(\Delta t)^{\alpha} \tau_{n}^{(k, i)}, \quad k \leq n \leq N .
$$

Multiplying $\xi^{n-k}$ on both sides of (4.4) and summing up for all $n \geq k$, we obtain

$$
\begin{aligned}
& \sum_{n=0}^{\infty} \sum_{m=0}^{k-1}\left(w_{n+k, m}^{(k, i)}+\omega_{n+k-m}^{(k, i)}\right) e_{m}^{(k, i)} \xi^{n}+\sum_{n=0}^{\infty} \sum_{j=k}^{n+k} \omega_{n+k-j}^{(k, i)} e_{j}^{(k, i)} \xi^{n} \\
& =(\Delta t)^{\alpha} \sum_{n=0}^{\infty} \delta f_{n+k}^{(k, i)} \xi^{n}+(\Delta t)^{\alpha} \sum_{n=0}^{\infty} \tau_{n+k}^{(k, i)} \xi^{n} .
\end{aligned}
$$

It follows that

$$
\omega^{(k, i)}(\xi) e^{(k, i)}(\xi)=\sum_{m=0}^{k-1} e_{m}^{(k, i)} s_{m}^{(k, i)}(\xi)+(\Delta t)^{\alpha} \delta f^{(k, i)}(\xi)+(\Delta t)^{\alpha} \tau^{(k, i)}(\xi),
$$


where

$$
\begin{aligned}
& s_{m}^{(k, i)}(\xi):=\sum_{n=0}^{\infty} s_{n, m}^{(k, i)} \xi^{n}=-\sum_{n=0}^{\infty}\left(w_{n+k, m}^{(k, i)}+\omega_{n+k-m}^{(k, i)}\right) \xi^{n}, \quad e^{(k, i)}(\xi)=\sum_{n=0}^{\infty} e_{n+k}^{(k, i)} \xi^{n}, \\
& \omega^{(k, i)}(\xi)=\sum_{n=0}^{\infty} \omega_{n}^{(k, i)} \xi^{n}, \quad \delta f^{(k, i)}(\xi)=\sum_{n=0}^{\infty} \delta f_{n+k}^{(k, i)} \xi^{n}, \quad \tau^{(k, i)}(\xi)=\sum_{n=0}^{\infty} \tau_{n+k}^{(k, i)} \xi^{n} .
\end{aligned}
$$

Theorem 4.1 Let $u(t)$ and $u_{n}, k \leq n \leq N$ be the solutions of Eqs. (1.1) and (4.4), respectively. Assume that $f(t, u(t))$ in (1.1) satisfies the Lipschitz continuous condition with respect to $u$. If $u(t) \in C^{k+1}[0, T]$, then

(i) for $1 \leq k \leq 3$,

$$
\left|e_{n}^{(k, k)}\right| \leq C^{(k, k)}\left(\sum_{m=0}^{k-1}\left|e_{m}^{(k, k)}\right|+(\Delta t)^{(k+1)}+(\Delta t)^{k+1-\alpha} t_{n-1}^{\alpha}\right), \quad k \leq n \leq N,
$$

(ii) for $1 \leq i<k \leq 3$,

$$
\left|e_{n}^{(k, i)}\right| \leq C^{(k, i)}\left(\sum_{m=0}^{k-1}\left|e_{m}^{(k, i)}\right|+(\Delta t)^{k}+(\Delta t)^{k+1-\alpha} t_{n-1}^{\alpha}\right), \quad k \leq n \leq N,
$$

where $\Delta t>0$ is sufficiently small, $N \Delta t=T$, and $C^{(k, i)}>0$ are independent of $N$ and $n$.

Proof Substituting formula (B.14) into (4.5), and using (B.21), we have

$$
e^{(k, i)}(\xi)=\frac{r^{(k, i)}(\xi)}{(1-\xi)^{\alpha}}\left(\sum_{m=0}^{k-1} e_{m}^{(k, i)} s_{m}^{(k, i)}(\xi)+(\Delta t)^{\alpha} \delta f^{(k, i)}(\xi)+(\Delta t)^{\alpha} \tau^{(k, i)}(\xi)\right) .
$$

For $k \leq n \leq N$, we rewrite (4.9) in the equivalent form

$$
\begin{aligned}
e_{n}^{(k, i)}= & \sum_{m=0}^{k-1} e_{m}^{(k, i)} \sum_{j=0}^{n-k} r_{n-k-j}^{(k, i)} \sum_{i=0}^{j} g_{j-i}^{(-\alpha)} s_{i, m}^{(k, i)}+(\Delta t)^{\alpha} \sum_{j=0}^{n-k} r_{n-k-j}^{(k, i)} \sum_{i=0}^{j} g_{j-i}^{(-\alpha)} \delta f_{i+k}^{(k, i)} \\
& +(\Delta t)^{\alpha} \sum_{j=0}^{n-k} r_{n-k-j}^{(k, i)} \sum_{i=0}^{j} g_{j-i}^{(-\alpha)} \tau_{i+k}^{(k, i)}
\end{aligned}
$$

where coefficients $g_{n}^{(-\alpha)}$ are given in Lemma B.2. Since $f(t, u(t))$ satisfies the Lipschitz continuous condition by assumption, there exist constants $L^{(k, i)}>0$ such that $\left|\delta f_{n}^{(k, i)}\right| \leq$ $L^{(k, i)}\left|e_{n}^{(k, i)}\right|$ for $k \leq n \leq N$. It follows that

$$
\begin{aligned}
\left|e_{n}^{(k, i)}\right| \leq & \sum_{m=0}^{k-1}\left|e_{m}^{(k, i)}\right| \sum_{j=0}^{n-k}\left|r_{n-k-j}^{(k, i)}\right| \sum_{i=0}^{j} g_{j-i}^{(-\alpha)}\left|s_{i, m}^{(k, i)}\right| \\
& +(\Delta t)^{\alpha} \sum_{j=0}^{n-k} g_{n-k-j}^{(-\alpha)} \sum_{i=0}^{j}\left|r_{j-i}^{(k, i)}\right|\left(L^{(k, i)}\left|e_{i+k}^{(k, i)}\right|+\left|\tau_{i+k}^{(k, i)}\right|\right) .
\end{aligned}
$$


On the one hand, by (B.11) and (B.20), there exist constants $\tilde{c}_{k, i}>0$, such that $\left|s_{n, 0}^{(k, i)}\right| \leq$ $c_{k, i} \frac{n^{-\alpha}}{\Gamma(1-\alpha)} \leq \tilde{c}_{k, i} g_{n}^{(\alpha-1)}$. Hence, we obtain

$$
\begin{aligned}
\sum_{j=0}^{n-k}\left|r_{n-k-j}^{(k, i)}\right| \sum_{i=0}^{j} g_{j-i}^{(-\alpha)}\left|s_{i, 0}^{(k, i)}\right| & \leq \tilde{c}_{k, i} \sum_{j=0}^{n-k}\left|r_{n-k-j}^{(k, i)}\right| \sum_{i=0}^{j} g_{j-i}^{(-\alpha)} g_{i}^{(\alpha-1)} \\
& \leq \tilde{c}_{k, i} \sum_{j=0}^{\infty}\left|r_{j}^{(k, i)}\right|=\tilde{c}_{k, i} M_{\alpha}^{(k, i)}
\end{aligned}
$$

where $\sum_{i=0}^{j} g_{j-i}^{(-\alpha)} g_{i}^{(\alpha-1)}=1$ for any $j \geq 0$ in view of the identity $(1-\xi)^{-\alpha}(1-\xi)^{\alpha-1}=$ $(1-\xi)^{-1}$. On the other hand, there exist constants $\tilde{c}_{m}^{(k, i)}>0, m \geq 1$, such that $\left|s_{n, m}^{(k, i)}\right| \leq$ $c_{m}^{(k, i)} \frac{n^{-\alpha-1}}{|\Gamma(-\alpha)|} \leq \tilde{c}_{m}^{(k, i)}\left|g_{n}^{(\alpha)}\right|$. This gives

$$
\begin{aligned}
\sum_{j=0}^{n-k}\left|r_{n-k-j}^{(k, i)}\right| \sum_{l=0}^{j} g_{j-l}^{(-\alpha)}\left|s_{l, m}^{(k, i)}\right| & \leq \tilde{c}_{m}^{(k, i)} \sum_{j=0}^{n-k}\left|r_{n-k-j}^{(k, i)}\right| \sum_{l=0}^{j} g_{j-l}^{(-\alpha)}\left|g_{l}^{(\alpha)}\right| \\
& \leq 2 \tilde{c}_{m}^{(k, i)} \sum_{j=0}^{n-k}\left|r_{n-k-j}^{(k, i)}\right| g_{j}^{(-\alpha)},
\end{aligned}
$$

where the last inequality holds since it is satisfied that $\sum_{l=0}^{j} g_{j-l}^{(-\alpha)} g_{l}^{(\alpha)}=0$ for any $j \geq 1$, and $\sum_{l=0}^{j} g_{j-l}^{(-\alpha)}\left|g_{l}^{(\alpha)}\right|=g_{j}^{(-\alpha)} g_{0}^{(\alpha)}-\sum_{l=1}^{j} g_{j-l}^{(-\alpha)} g_{l}^{(\alpha)}=2 g_{j}^{(-\alpha)}$ follows from Lemma B.2. In addition, the sequences $\left\{r_{n}^{(k, i)}\right\}$ belong to $l^{1}$, and $g_{n}^{(-\alpha)} \rightarrow 0$ as $n \rightarrow \infty$. Therefore, $\sum_{j=0}^{n-k}\left|r_{n-k-j}^{(k, i)}\right| g_{j}^{(-\alpha)} \rightarrow 0$ as $n \rightarrow \infty$. Then, the sequences $\sum_{j=0}^{n-k}\left|r_{n-k-j}^{(k, i)}\right| \sum_{l=0}^{j} g_{j-l}^{(-\alpha)}\left|s_{l, m}^{(k, i)}\right|$ can be bounded by $2 \tilde{c}_{m}^{(k, i)} M_{\alpha}^{(k, i)}$.

In the cases $1 \leq k \leq 3$, recalling $\left|\tau_{n}^{(k, k)}\right| \leq C_{\alpha}^{(k)}(\Delta t)^{k+1-\alpha}$ uniformly for $n \geq k$ in Theorem 2.1, together with (B.17), we have

$$
\begin{aligned}
(\Delta t)^{\alpha} \sum_{j=0}^{n-k}\left|r_{n-k-j}^{(k, k)}\right| \sum_{i=0}^{j} g_{j-i}^{(-\alpha)}\left|\tau_{i+k}^{(k, k)}\right| & =(\Delta t)^{\alpha} \sum_{j=0}^{n-k} g_{n-k-j}^{(-\alpha)} \sum_{i=0}^{j}\left|r_{j-i}^{(k, k)}\right|\left|\tau_{i+k}^{(k, k)}\right| \\
& \leq(\Delta t)^{k+1} C_{\alpha}^{(k)} M_{\alpha}^{(k, k)} \sum_{j=0}^{n-k} g_{j}^{(-\alpha)} \\
& \leq(\Delta t)^{k+1} C_{\alpha}^{(k)} M_{\alpha}^{(k, k)}\left(1+C \sum_{j=1}^{n-k} \frac{j^{\alpha-1}}{\Gamma(\alpha)}\right) \\
& \leq(\Delta t)^{k+1} C_{\alpha}^{(k)} M_{\alpha}^{(k, k)}\left(1+\frac{C}{\Gamma(\alpha)} \int_{0}^{n-k} t^{\alpha-1} \mathrm{~d} t\right) \\
& \leq \tilde{C}_{\alpha}^{(k, k)}\left((\Delta t)^{k+1}+(\Delta t)^{k+1-\alpha} t_{n-k}^{\alpha}\right)
\end{aligned}
$$

In other cases $1 \leq i<k \leq 3$, according to Theorem 2.1, there exist constants $C_{\alpha}^{(k, i)}>0$, such that

$$
\left|\tau_{n}^{(k, i)}\right| \leq C_{\alpha}^{(k, i)}\left((\Delta t)^{k-\alpha} \frac{(n-k)^{-\alpha-1}}{|\Gamma(-\alpha)|}+\frac{(\Delta t)^{k+1-\alpha}}{\Gamma(1-\alpha)}\right), \quad n \geq k .
$$


Together with (B.17), it follows that

$$
\begin{aligned}
(\Delta t)^{\alpha} \sum_{j=0}^{n-k}\left|r_{n-k-j}^{(k, i)}\right| \sum_{l=0}^{j} g_{j-l}^{(-\alpha)}\left|\tau_{l+k}^{(k, i)}\right| \leq & C_{\alpha}^{(k, i)}\left((\Delta t)^{k} \tilde{c}_{\alpha} \sum_{j=0}^{n-k}\left|r_{n-k-j}^{(k, i)}\right| \sum_{l=0}^{j} g_{j-l}^{(-\alpha)}\left|g_{l}^{(\alpha)}\right|\right. \\
& \left.+(\Delta t)^{k+1} \sum_{j=0}^{n-k}\left|r_{n-k-j}^{(k, i)}\right| \sum_{l=0}^{n-k} g_{l}^{(-\alpha)}\right) \\
\leq & C_{\alpha}^{(k, i)}\left(2(\Delta t)^{k} \tilde{c}_{\alpha} \sum_{j=0}^{n-k}\left|r_{n-k-j}^{(k, i)}\right| g_{j}^{(-\alpha)}\right. \\
& \left.+(\Delta t)^{k+1} \sum_{j=0}^{n-k}\left|r_{n-k-j}^{(k, i)}\right| g_{n-k}^{(-\alpha-1)}\right) \\
\leq & \tilde{C}_{\alpha}^{(k, i)}\left((\Delta t)^{k}+(\Delta t)^{k+1-\alpha} t_{n-k}^{\alpha}\right)
\end{aligned}
$$

Therefore formula (4.11) becomes

$$
\begin{aligned}
\left|e_{n}^{(k, i)}\right| \leq & (\Delta t)^{\alpha} L^{(k, i)}\left(\sum_{j=0}^{n-k-1} g_{n-k-j}^{(-\alpha)} \sum_{l=0}^{j}\left|r_{j-l}^{(k, i)}\left\|e_{l+k}^{(k, i)}\left|+g_{0}^{(-\alpha)} \sum_{l=0}^{n-k-1}\right| r_{n-k-l}^{(k, i)}\right\| e_{l+k}^{(k, i)}\right|\right. \\
& \left.+g_{0}^{(-\alpha)}\left|r_{0}^{(k, i)}\right|\left|e_{n}^{(k, i)}\right|\right)+\delta_{n}^{(k, i)}, \quad n \geq k .
\end{aligned}
$$

For $1 \leq i \leq k \leq 3$, we obtain from (4.12), (4.13), (4.14) and (4.15) that

$$
\delta_{n}^{(k, k)}=C_{\alpha}^{(k, k)}\left(\sum_{m=0}^{k-1}\left|e_{m}^{(k, k)}\right|+(\Delta t)^{(k+1)}+(\Delta t)^{k+1-\alpha} t_{n-1}^{\alpha}\right), \quad n \geq k
$$

and

$$
\delta_{n}^{(k, i)}=C_{\alpha}^{(k, i)}\left(\sum_{m=0}^{k-1}\left|e_{m}^{(k, i)}\right|+(\Delta t)^{k}+(\Delta t)^{k+1-\alpha} t_{n-1}^{\alpha}\right), \quad n \geq k,
$$

where $C_{\alpha}^{(k, i)}=\max \left\{\tilde{c}_{k, i} M_{\alpha}^{(k, i)}, 2 \tilde{c}_{m}^{(k, i)} M_{\alpha}^{(k, i)}, \tilde{C}_{\alpha}^{(k, i)}\right\}$. Let $\Delta t>0$ be sufficiently small. Then there exist bounded constants $c_{k, i}^{*}$ such that $0<\frac{1}{1-(\Delta t)^{\alpha} L^{(k, i)} g_{0}^{(-\alpha)}\left|r_{0}^{(k, i)}\right|} \leq c_{k, i}^{*}$, and

$$
\left\{\begin{aligned}
\left|e_{k}^{(k, i)}\right| \leq & \tilde{\delta}_{k}^{(k, i)}, \\
\left|e_{n}^{(k, i)}\right| \leq & \tilde{\delta}_{n}^{(k, i)}+(\Delta t)^{\alpha} c_{k, i}^{*} L^{(k, i)}\left(\sum_{j=0}^{n-k-1} g_{n-k-j}^{(-\alpha)} \sum_{l=0}^{j}\left|r_{j-l}^{(k, i)}\right|\left|e_{l+k}^{(k, i)}\right|\right. \\
& \left.+g_{0}^{(-\alpha)} \sum_{l=0}^{n-k-1}\left|r_{n-k-l}^{(k, i)}\right|\left|e_{l+k}^{(k, i)}\right|\right), \quad n \geq k+1
\end{aligned}\right.
$$


where $\tilde{\delta}_{n}^{(k, i)}=c_{k, i}^{*} \delta_{n}^{(k, i)}$. Next, assume that $\left\{p_{n}^{(k, i)}\right\}_{n \geq 0}$ are a series of non-negative sequences satisfying

$$
\left\{\begin{array}{l}
p_{0}^{(k, i)}=\tilde{\delta}_{k}^{(k, i)}, \\
p_{n}^{(k, i)}=\tilde{\delta}_{n+k}^{(k, i)}+\frac{(\Delta t)^{\alpha} \tilde{L}^{(k, i)}}{\Gamma(\alpha)} \sum_{j=0}^{n-1}(n-j)^{\alpha-1} p_{j}^{(k, i)}, \quad n \geq 1,
\end{array}\right.
$$

where $\tilde{L}^{(k, i)}$ are chosen such that

$$
\tilde{L}^{(k, i)}=\max \left\{c_{k, i}^{*} L^{(k, i)} M_{\alpha}^{(k, i)}\left(1+\Gamma(\alpha) g_{1}^{(-\alpha)}\right), c_{k, i}^{*} L^{(k, i)} M_{\alpha}^{(k, i)} g_{n}^{(-\alpha)} n^{1-\alpha} \Gamma(\alpha)\right\} .
$$

Then using the weakly singular discrete Gronwall inequality in [14], we conclude that $\left\{p_{n}^{(k, i)}\right\}_{n \geq 1}$ is monotonically increasing with respect to $n$, and satisfy

$$
p_{n}^{(k, i)} \leq \tilde{\delta}_{n+k}^{(k, i)} E_{\alpha}\left(\tilde{L}^{(k, i)}(n \Delta t)^{\alpha}\right), \quad n \geq 1,
$$

where $E_{\alpha}(\cdot)$ denote Mittag-Leffler functions. In addition, from (4.16) and (4.17), we have $\left|e_{n}^{(k, i)}\right| \leq p_{n-k}^{(k, i)}$ for $n \geq k$, and consequently,

$$
\left|e_{n}^{(k, i)}\right| \leq \tilde{\delta}_{n}^{(k, i)} E_{\alpha}\left(\tilde{L}^{(k, i)}(n-k)^{\alpha} \Delta t^{\alpha}\right) \leq \tilde{\delta}_{n}^{(k, i)} E_{\alpha}\left(\tilde{L}^{(k, i)} T^{\alpha}\right)
$$

for $k \leq n \leq N$.

Remark 3 Note that the error estimates (4.7) and (4.8) are uniform for all $n \geq k$. For those $t_{n}$ away from the origin, under the conditions $e_{m}^{(k, i)}=O\left((\Delta t)^{k}\right)$ for $1 \leq m \leq k-1$, we can observe that the errors are $(k+1-\alpha)$-th order accurate in time in the cases $1 \leq i \leq k \leq 3$.

\section{Numerical Experiments}

In this section, we utilize (2.10) to approximate the equations in Examples 5.1 and 5.2, and prescribe starting values exactly.

Example 5.1 Consider the linear fractional ordinary differential equation

$$
\left\{\begin{array}{l}
{ }^{C} D^{\alpha} u(t)=\lambda u(t)+f(t), \quad t \in(0,1], \\
u(0)=u_{0},
\end{array}\right.
$$

where $0<\alpha<1$. The exact solution is given by $u(t)=e^{-t} \in C^{\infty}[0,1]$, if $f(t)=$ $-t^{1-\alpha} E_{1,2-\alpha}(-t)-\lambda e^{-t} \in C[0,1] \cap C^{\infty}(0,1]$, where the Mittag-Leffler functions [36] are defined by

$$
E_{\alpha, \beta}(t)=\sum_{k=0}^{\infty} \frac{t^{k}}{\Gamma(\alpha k+\beta)}, \quad \alpha>0, \beta>0
$$

In Fig. 1a-d, we plot the truncated boundary locus curves $\sum_{n=0}^{6000} \omega_{n}^{(k, i)} e^{\sqrt{-1} \theta n}(0 \leq \theta \leq$ $2 \pi)$ for $1 \leq i \leq k \leq 3$ and some $\alpha \in(0,1)$. It is already known from Theorem 3.1 that the stability regions of methods (3.2) lie outside their boundary locus curves. Here, we introduce the points $z_{n}=\lambda\left(\Delta t_{n}\right)^{\alpha}, 1 \leq n \leq 5$, where $\Delta t_{n}=1 / 2^{n+6}$ denote different time steps. Tables 1 and 2 show the accuracy and convergence rates of the error $\left|u\left(t_{M}\right)-u_{M}^{(k, i)}\right|$ for 


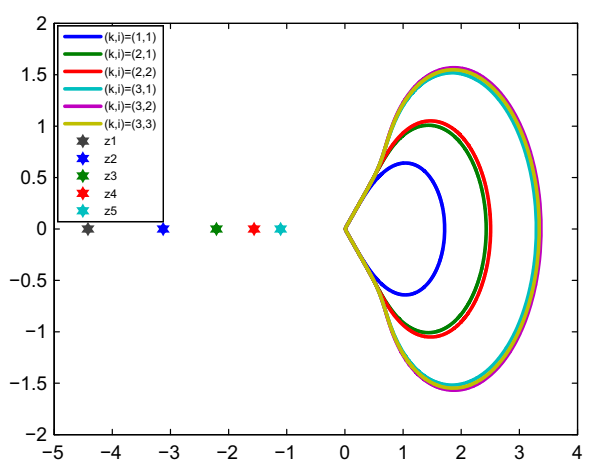

(a)

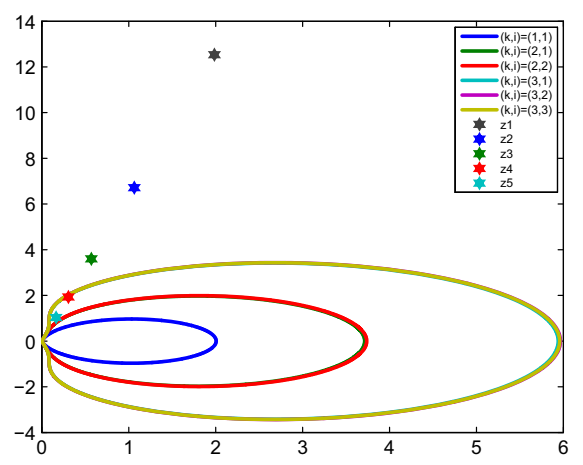

(c)

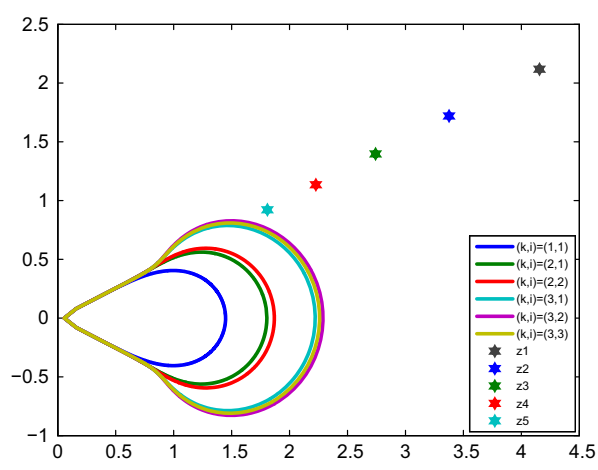

(b)

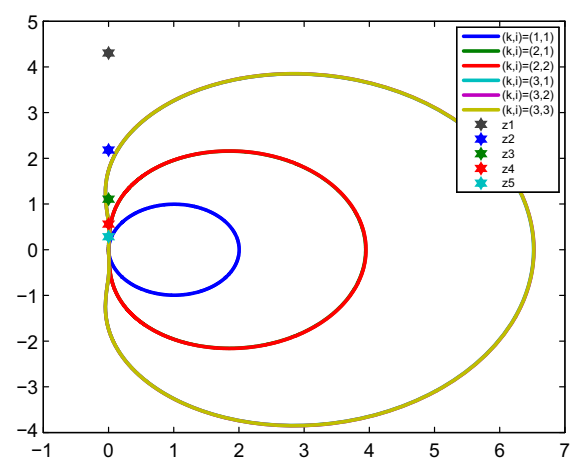

(d)

Fig. 1 The boundary of the stability region for different $\alpha$ and $\lambda \mathbf{a} \alpha=0.5, \lambda=-50, \mathbf{b} \alpha=0.3$, $\lambda=20 \times e^{\frac{i \pi \alpha}{2}}, \mathbf{c} \alpha=0.9, \lambda=1000 \times e^{\frac{i \pi \alpha}{2}}, \mathbf{d} \alpha=0.98, \lambda=500 i$

Example 5.1, where $t_{M}=1$ is fixed and $M=2^{j}$ for $7 \leq j \leq 11, u\left(t_{M}\right)$ and $u_{M}^{(k, i)}$ are the exact solution and computed solution for (5.1), respectively.

From Fig. 1a-d and Tables 1, 2, we can see the influence of the stability of a numerical method on global error. In Fig. 1a, the points $z_{n}$ with $1 \leq n \leq 5$ all lie in the stability regions for $\alpha=0.5$ and $\lambda=-50$, we get $(k+1-\alpha)$-th order of accuracy shown in Tables 1,2 . In Fig. 1b, c, $\left\{z_{n}\right\}_{n=1}^{5}$ fall on the half line with angle $\frac{\pi \alpha}{2}$. It is observed that when all $\left\{z_{n}\right\}_{n=1}^{5}$ fall out of the instability region (cf. Fig. 1b), correspondingly, as shown in Tables 1, 2, the global error agrees with $(k+1-\alpha)$-th order of accuracy. On the other hand, due to the points $z_{4}$ and $z_{5}$ outside the stability regions for $k=3$ (cf. Fig. 1c), perturbation errors are magnified and accumulated significantly, which are shown in Tables 1, 2 as well. In Fig. 1d, $\left\{z_{n}\right\}$ are chosen on the imaginary axis with pure imaginary number $\lambda$, Theorem 3.2 tells us that all $\left\{z_{n}\right\}$ are in the stability region for $k=1,2$. The error and convergence order are obtained (cf. Table 1).

As a counter example, in Fig. 1d, the point $z_{3}$ doesn't belong to the stability region for $\alpha=0.98$, for this case, the errors shown in Table 2 blow up. In fact, it can be observed that for $k=3$, methods (3.2) don't possess $A\left(\frac{\pi}{2}\right)$-stability when $\alpha$ tends to 1 , as it is known that BDF3 method for ODEs is not $A\left(\frac{\pi}{2}\right)$-stable. 


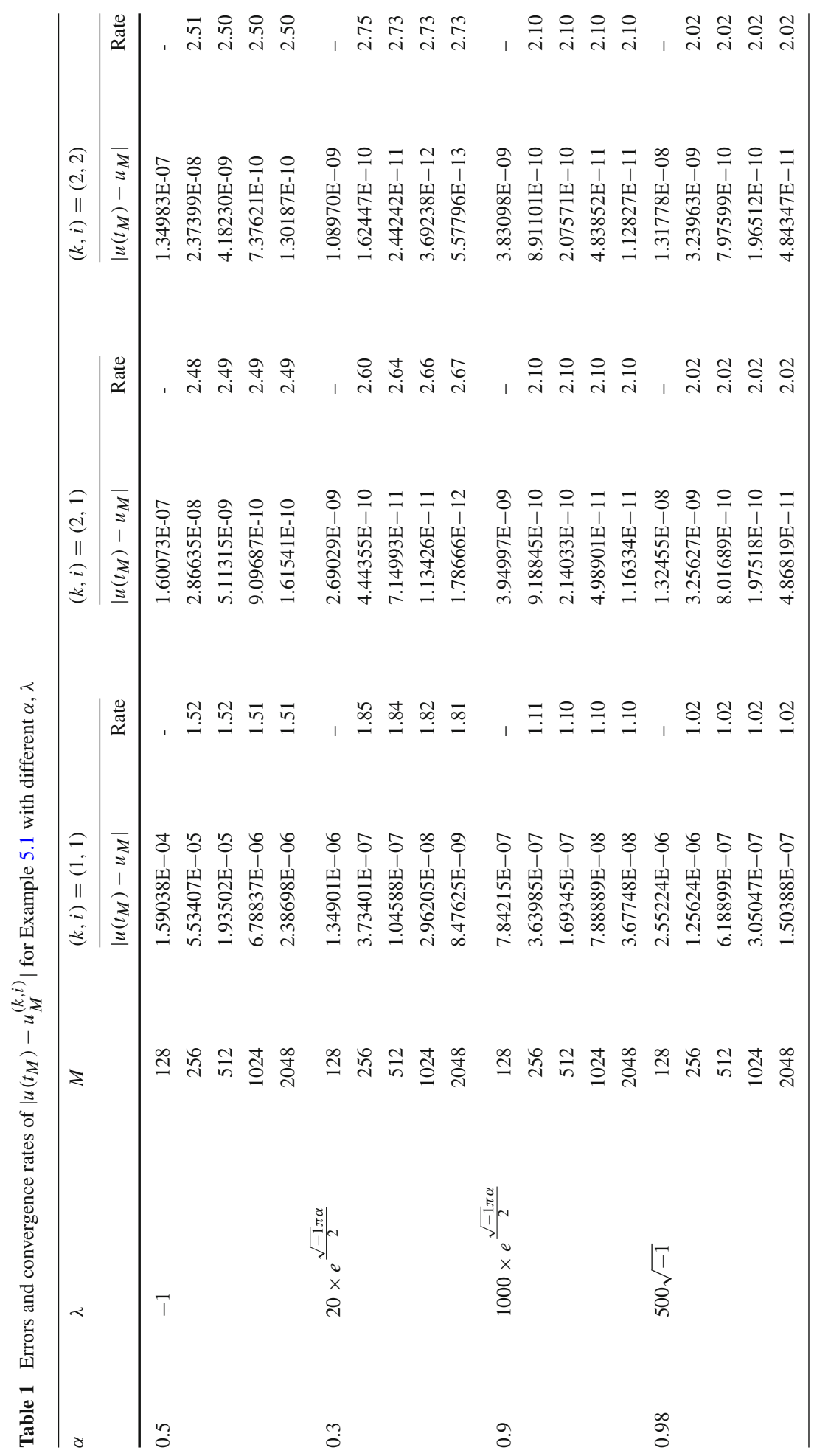




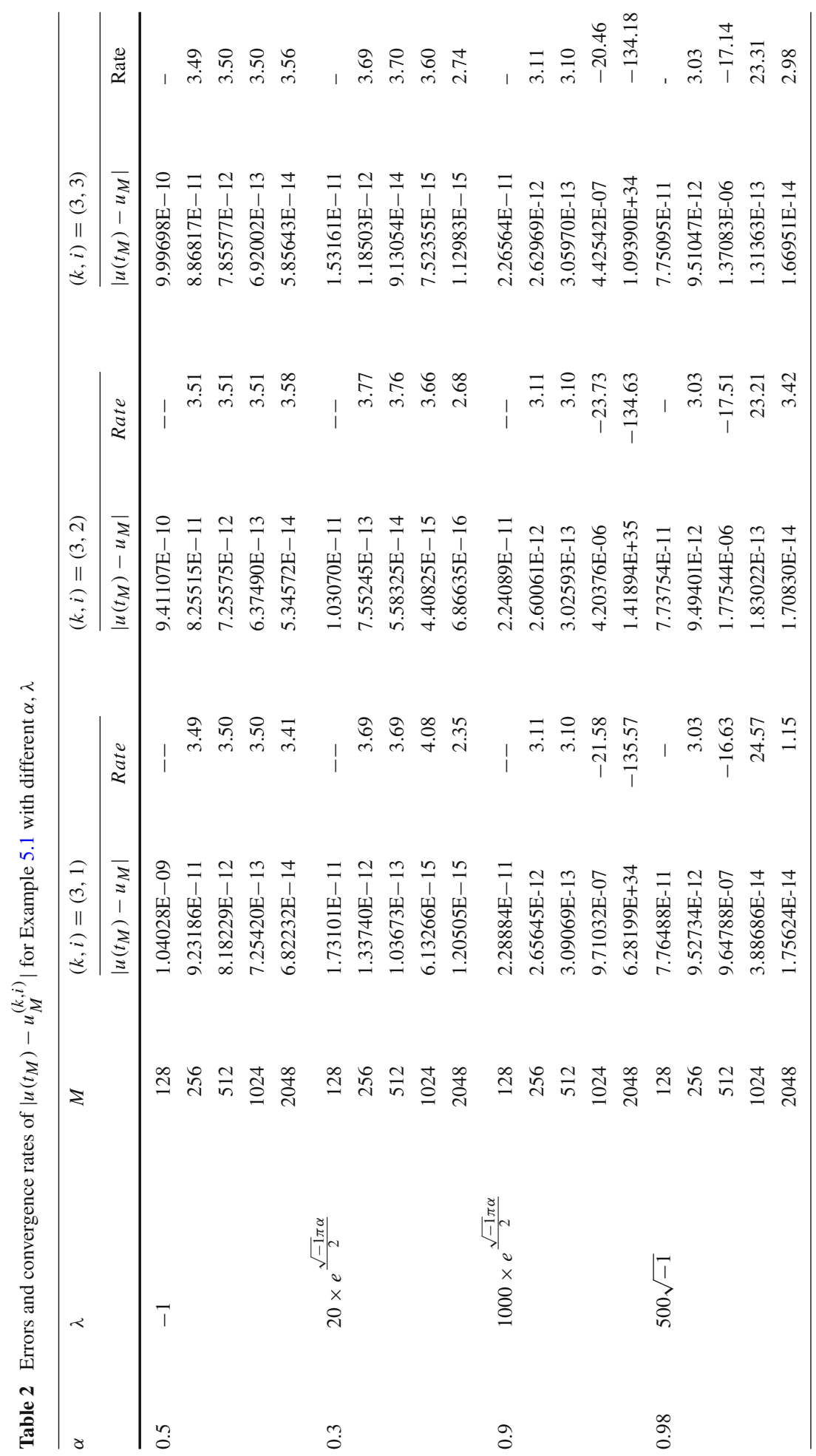


Table 3 Errors and convergence orders of $\left|u\left(t_{M}\right)-u_{M}^{(k, i)}\right|$ for Example 5.2 with $\mu=-1$

\begin{tabular}{|c|c|c|c|c|c|c|c|}
\hline \multirow[t]{2}{*}{$\alpha$} & \multirow[t]{2}{*}{ M } & \multicolumn{2}{|l|}{$(k, i)=(1,1)$} & \multicolumn{2}{|l|}{$(k, i)=(2,1)$} & \multicolumn{2}{|l|}{$(k, i)=(2,2)$} \\
\hline & & $\left|u\left(t_{M}\right)-u_{M}\right|$ & rate & $\left|u\left(t_{M}\right)-u_{M}\right|$ & rate & $\left|u\left(t_{M}\right)-u_{M}\right|$ & rate \\
\hline \multirow[t]{5}{*}{0.1} & 32 & $1.77420 \mathrm{E}-05$ & - & $1.34563 \mathrm{E}-07$ & - & $1.44620 \mathrm{E}-08$ & - \\
\hline & 64 & $5.02145 \mathrm{E}-06$ & 1.82 & $2.09471 \mathrm{E}-08$ & 2.68 & $5.84367 \mathrm{E}-10$ & 4.63 \\
\hline & 128 & $1.41283 \mathrm{E}-06$ & 1.83 & $3.17430 \mathrm{E}-09$ & 2.72 & $3.71249 \mathrm{E}-10$ & 0.65 \\
\hline & 256 & $3.95463 \mathrm{E}-07$ & 1.84 & $4.71557 \mathrm{E}-10$ & 2.75 & $8.48118 \mathrm{E}-11$ & 2.13 \\
\hline & 512 & $1.10196 \mathrm{E}-07$ & 1.84 & $6.89781 \mathrm{E}-11$ & 2.77 & $1.56365 \mathrm{E}-11$ & 2.44 \\
\hline \multirow[t]{5}{*}{0.3} & 32 & $1.02368 \mathrm{E}-04$ & - & $1.16950 \mathrm{E}-06$ & - & $7.44373 \mathrm{E}-07$ & - \\
\hline & 64 & $3.21104 \mathrm{E}-05$ & 1.67 & $1.92603 \mathrm{E}-07$ & 2.60 & $1.07277 \mathrm{E}-07$ & 2.79 \\
\hline & 128 & $1.00362 \mathrm{E}-05$ & 1.68 & $3.12510 \mathrm{E}-08$ & 2.62 & $1.57091 \mathrm{E}-08$ & 2.77 \\
\hline & 256 & $3.12764 \mathrm{E}-06$ & 1.68 & $5.01368 \mathrm{E}-09$ & 2.64 & $2.32621 \mathrm{E}-09$ & 2.76 \\
\hline & 512 & $9.72370 \mathrm{E}-07$ & 1.69 & $7.97379 \mathrm{E}-10$ & 2.65 & $3.47263 \mathrm{E}-10$ & 2.74 \\
\hline \multirow[t]{5}{*}{0.5} & 32 & $3.61074 \mathrm{E}-04$ & - & $5.29046 \mathrm{E}-06$ & - & $4.57737 \mathrm{E}-06$ & - \\
\hline & 64 & $1.28284 \mathrm{E}-04$ & 1.49 & $9.63089 \mathrm{E}-07$ & 2.46 & $7.97338 \mathrm{E}-07$ & 2.52 \\
\hline & 128 & $4.55113 \mathrm{E}-05$ & 1.50 & $1.73854 \mathrm{E}-07$ & 2.47 & $1.39694 \mathrm{E}-07$ & 2.51 \\
\hline & 256 & $1.61301 \mathrm{E}-05$ & 1.50 & $3.11964 \mathrm{E}-08$ & 2.48 & $2.45589 \mathrm{E}-08$ & 2.51 \\
\hline & 512 & $5.71284 \mathrm{E}-06$ & 1.50 & $5.57391 \mathrm{E}-09$ & 2.48 & $4.32640 \mathrm{E}-09$ & 2.51 \\
\hline \multirow[t]{5}{*}{0.7} & 32 & $1.13040 \mathrm{E}-03$ & - & $1.95934 \mathrm{E}-05$ & - & $1.88894 \mathrm{E}-05$ & - \\
\hline & 64 & $4.59858 \mathrm{E}-04$ & 1.30 & $4.02983 \mathrm{E}-06$ & 2.28 & $3.82681 \mathrm{E}-06$ & 2.30 \\
\hline & 128 & $1.86904 \mathrm{E}-04$ & 1.30 & $8.24864 \mathrm{E}-07$ & 2.29 & $7.76005 \mathrm{E}-07$ & 2.30 \\
\hline & 256 & $7.59344 \mathrm{E}-05$ & 1.30 & $1.68344 \mathrm{E}-07$ & 2.29 & $1.57456 \mathrm{E}-07$ & 2.30 \\
\hline & 512 & $3.08447 \mathrm{E}-05$ & 1.30 & $3.42933 \mathrm{E}-08$ & 2.30 & $3.19602 \mathrm{E}-08$ & 2.30 \\
\hline
\end{tabular}

Example 5.2 Consider the nonlinear equation

$$
\left\{\begin{array}{l}
{ }^{C} D^{\alpha} u(t)=-u^{2}+f(t), \quad t \in(0,1] \\
u(0)=u_{0} .
\end{array}\right.
$$

The source function is prescribed by $f(t)=\mu t^{1-\alpha} E_{1,2-\alpha}(\mu t)+e^{2 \mu t}$ such that the exact solution reads $u(t)=e^{\mu t}$.

We use (2.17) in combination with Newton's method for solving the nonlinear equation (5.2). Tables 3, 4, 5 and 6 show the global error $\left|e_{M}^{(k, i)}\right|=\left|u\left(t_{M}\right)-u_{M}^{(k, i)}\right|$ and orders of accuracy for Example 5.2 with different $\mu$ and $\alpha$, where $t_{M}=1$ is fixed and $\Delta t=1 / M$ with $M=2^{j}, 5 \leq j \leq 9$. Further, it is observed that $\left|e_{M}^{(k, i)}\right|=O\left(\Delta t^{k+1-\alpha}\right)$ for $1 \leq i \leq k \leq 3$.

\section{Conclusions}

We have proposed a class of new high-order approximations for solving time-fractional initial value models of order $0<\alpha<1$. Furthermore, the local truncation error estimate in terms of a smooth solution is presented. Additionally, stability and convergence analysis of these numerical methods are discussed in detail. This will promote further investigation of the proposed methods for solving time-fractional partial differential equations. 
Table 4 Errors and convergence orders of $\left|u\left(t_{M}\right)-u_{M}^{(k, i)}\right|$ for Example 5.2 with $\mu=-1$

\begin{tabular}{|c|c|c|c|c|c|c|c|}
\hline \multirow[t]{2}{*}{$\alpha$} & \multirow[t]{2}{*}{$M$} & \multicolumn{2}{|l|}{$(k, i)=(3,1)$} & \multicolumn{2}{|l|}{$(k, i)=(3,2)$} & \multicolumn{2}{|l|}{$(k, i)=(3,3)$} \\
\hline & & $\left|u\left(t_{M}\right)-u_{M}\right|$ & rate & $\left|u\left(t_{M}\right)-u_{M}\right|$ & rate & $\left|u\left(t_{M}\right)-u_{M}\right|$ & rate \\
\hline \multirow[t]{5}{*}{0.1} & 32 & $5.97442 \mathrm{E}-09$ & - & $4.12374 \mathrm{E}-09$ & - & $5.22481 \mathrm{E}-09$ & - \\
\hline & 64 & $4.22608 \mathrm{E}-10$ & 3.82 & $2.53599 \mathrm{E}-10$ & 4.02 & $3.70957 \mathrm{E}-10$ & 3.82 \\
\hline & 128 & $2.96461 \mathrm{E}-11$ & 3.83 & $1.56801 \mathrm{E}-11$ & 4.02 & $2.61735 \mathrm{E}-11$ & 3.83 \\
\hline & 256 & $2.06629 \mathrm{E}-12$ & 3.84 & $9.72611 \mathrm{E}-13$ & 4.01 & $1.83664 \mathrm{E}-12$ & 3.83 \\
\hline & 512 & $1.51434 \mathrm{E}-13$ & 3.77 & $5.90084 \mathrm{E}-14$ & 4.04 & $1.25844 \mathrm{E}-13$ & 3.87 \\
\hline \multirow[t]{5}{*}{0.3} & 32 & $3.63586 \mathrm{E}-08$ & - & $2.98609 \mathrm{E}-08$ & - & $3.32377 \mathrm{E}-08$ & - \\
\hline & 64 & $2.85491 \mathrm{E}-09$ & 3.67 & $2.18534 \mathrm{E}-09$ & 3.77 & $2.60248 \mathrm{E}-09$ & 3.67 \\
\hline & 128 & $2.22890 \mathrm{E}-10$ & 3.68 & $1.61522 \mathrm{E}-10$ & 3.76 & $2.03176 \mathrm{E}-10$ & 3.68 \\
\hline & 256 & $1.73471 \mathrm{E}-11$ & 3.68 & $1.20285 \mathrm{E}-11$ & 3.75 & $1.58175 \mathrm{E}-11$ & 3.68 \\
\hline & 512 & $1.35003 \mathrm{E}-12$ & 3.68 & $9.01168 \mathrm{E}-13$ & 3.74 & $1.22508 \mathrm{E}-12$ & 3.69 \\
\hline \multirow[t]{5}{*}{0.5} & 32 & $1.38110 \mathrm{E}-07$ & - & $1.27455 \mathrm{E}-07$ & - & $1.32024 \mathrm{E}-07$ & - \\
\hline & 64 & $1.23087 \mathrm{E}-08$ & 3.49 & $1.10139 \mathrm{E}-08$ & 3.53 & $1.17199 \mathrm{E}-08$ & 3.49 \\
\hline & 128 & $1.09270 \mathrm{E}-09$ & 3.49 & $9.57697 \mathrm{E}-10$ & 3.52 & $1.03891 \mathrm{E}-09$ & 3.50 \\
\hline & 256 & $9.68335 \mathrm{E}-11$ & 3.50 & $8.36696 \mathrm{E}-11$ & 3.52 & $9.20156 \mathrm{E}-11$ & 3.50 \\
\hline & 512 & $8.57364 \mathrm{E}-12$ & 3.50 & $7.33291 \mathrm{E}-12$ & 3.51 & $8.14260 \mathrm{E}-12$ & 3.50 \\
\hline \multirow[t]{5}{*}{0.7} & 32 & $4.71501 \mathrm{E}-07$ & - & $4.61657 \mathrm{E}-07$ & - & $4.64497 \mathrm{E}-07$ & - \\
\hline & 64 & $4.82158 \mathrm{E}-08$ & 3.29 & $4.66544 \mathrm{E}-08$ & 3.31 & $4.73668 \mathrm{E}-08$ & 3.29 \\
\hline & 128 & $4.91018 \mathrm{E}-09$ & 3.30 & $4.71769 \mathrm{E}-09$ & 3.31 & $4.81794 \mathrm{E}-09$ & 3.30 \\
\hline & 256 & $4.99217 \mathrm{E}-10$ & 3.30 & $4.77606 \mathrm{E}-10$ & 3.30 & $4.89591 \mathrm{E}-10$ & 3.30 \\
\hline & 512 & $5.07119 \mathrm{E}-11$ & 3.30 & $4.84126 \mathrm{E}-11$ & 3.30 & $4.97420 \mathrm{E}-11$ & 3.30 \\
\hline
\end{tabular}

Table 5 Errors and convergence orders of $\left|u\left(t_{M}\right)-u_{M}^{(k, i)}\right|$ for Example 5.2 with $\mu=\sqrt{-1}$

\begin{tabular}{|c|c|c|c|c|c|c|c|}
\hline \multirow[t]{2}{*}{$\alpha$} & \multirow[t]{2}{*}{$M$} & \multicolumn{2}{|l|}{$(k, i)=(1,1)$} & \multicolumn{2}{|l|}{$(k, i)=(2,1)$} & \multicolumn{2}{|l|}{$(k, i)=(2,2)$} \\
\hline & & $\left|u\left(t_{M}\right)-u_{M}\right|$ & Rate & $\left|u\left(t_{M}\right)-u_{M}\right|$ & Rate & $\left|u\left(t_{M}\right)-u_{M}\right|$ & Rate \\
\hline \multirow[t]{5}{*}{0.1} & 32 & $2.54121 \mathrm{E}-05$ & - & $3.52358 \mathrm{E}-07$ & - & $8.56779 \mathrm{E}-08$ & - \\
\hline & 64 & $7.32713 \mathrm{E}-06$ & 1.79 & $5.14268 \mathrm{E}-08$ & 2.78 & $8.08705 \mathrm{E}-09$ & 3.41 \\
\hline & 128 & $2.09321 \mathrm{E}-06$ & 1.81 & $7.42653 \mathrm{E}-09$ & 2.79 & $7.38063 \mathrm{E}-10$ & 3.45 \\
\hline & 256 & $5.93428 \mathrm{E}-07$ & 1.82 & $1.06282 \mathrm{E}-09$ & 2.80 & $8.51842 \mathrm{E}-11$ & 3.12 \\
\hline & 512 & $1.67161 \mathrm{E}-07$ & 1.83 & $1.50938 \mathrm{E}-10$ & 2.82 & $1.47554 \mathrm{E}-11$ & 2.53 \\
\hline \multirow[t]{5}{*}{0.3} & 32 & $1.44121 \mathrm{E}-04$ & - & $2.21534 \mathrm{E}-06$ & - & $1.22445 \mathrm{E}-06$ & - \\
\hline & 64 & $4.58574 \mathrm{E}-05$ & 1.65 & $3.56433 \mathrm{E}-07$ & 2.64 & $1.77215 \mathrm{E}-07$ & 2.79 \\
\hline & 128 & $1.44905 \mathrm{E}-05$ & 1.66 & $5.68067 \mathrm{E}-08$ & 2.65 & $2.58224 \mathrm{E}-08$ & 2.78 \\
\hline & 256 & $4.55432 \mathrm{E}-06$ & 1.67 & $8.98792 \mathrm{E}-09$ & 2.66 & $3.78933 \mathrm{E}-09$ & 2.77 \\
\hline & 512 & $1.42539 \mathrm{E}-06$ & 1.68 & $1.41404 \mathrm{E}-09$ & 2.67 & $5.59864 \mathrm{E}-10$ & 2.76 \\
\hline \multirow[t]{5}{*}{0.5} & 32 & $4.89012 \mathrm{E}-04$ & - & $8.26670 \mathrm{E}-06$ & - & $6.43523 \mathrm{E}-06$ & - \\
\hline & 64 & $1.75193 \mathrm{E}-04$ & 1.48 & $1.49308 \mathrm{E}-06$ & 2.47 & $1.12324 \mathrm{E}-06$ & 2.52 \\
\hline & 128 & $6.25162 \mathrm{E}-05$ & 1.49 & $2.67884 \mathrm{E}-07$ & 2.48 & $1.96610 \mathrm{E}-07$ & 2.51 \\
\hline & 256 & $2.22470 \mathrm{E}-05$ & 1.49 & $4.78471 \mathrm{E}-08$ & 2.49 & $3.44997 \mathrm{E}-08$ & 2.51 \\
\hline & 512 & $7.90153 \mathrm{E}-06$ & 1.49 & $8.51955 \mathrm{E}-09$ & 2.49 & $6.06569 \mathrm{E}-09$ & 2.51 \\
\hline
\end{tabular}


Table 5 continued

\begin{tabular}{|c|c|c|c|c|c|c|c|}
\hline \multirow[t]{2}{*}{$\alpha$} & \multirow[t]{2}{*}{$M$} & \multicolumn{2}{|l|}{$(k, i)=(1,1)$} & \multicolumn{2}{|l|}{$(k, i)=(2,1)$} & \multicolumn{2}{|l|}{$(k, i)=(2,2)$} \\
\hline & & $\left|u\left(t_{M}\right)-u_{M}\right|$ & Rate & $\left|u\left(t_{M}\right)-u_{M}\right|$ & Rate & $\left|u\left(t_{M}\right)-u_{M}\right|$ & Rate \\
\hline \multirow[t]{5}{*}{0.7} & 32 & $1.47058 \mathrm{E}-03$ & - & $2.70727 \mathrm{E}-05$ & - & $2.47625 \mathrm{E}-05$ & - \\
\hline & 64 & $6.00118 \mathrm{E}-04$ & 1.29 & $5.55402 \mathrm{E}-06$ & 2.29 & $5.02488 \mathrm{E}-06$ & 2.30 \\
\hline & 128 & $2.44431 \mathrm{E}-04$ & 1.30 & $1.13475 \mathrm{E}-06$ & 2.29 & $1.01945 \mathrm{E}-06$ & 2.30 \\
\hline & 256 & $9.94448 \mathrm{E}-05$ & 1.30 & $2.31281 \mathrm{E}-07$ & 2.29 & $2.06856 \mathrm{E}-07$ & 2.30 \\
\hline & 512 & $4.04305 \mathrm{E}-05$ & 1.30 & $4.70707 \mathrm{E}-08$ & 2.30 & $4.19810 \mathrm{E}-08$ & 2.30 \\
\hline
\end{tabular}

Table 6 Errors and convergence orders of $\left|u\left(t_{M}\right)-u_{M}^{(k, i)}\right|$ for Example 5.2 with $\mu=\sqrt{-1}$

\begin{tabular}{|c|c|c|c|c|c|c|c|}
\hline \multirow[t]{2}{*}{$\alpha$} & \multirow[t]{2}{*}{$M$} & \multicolumn{2}{|l|}{$(k, i)=(3,1)$} & \multicolumn{2}{|l|}{$(k, i)=(3,2)$} & \multicolumn{2}{|l|}{$(k, i)=(3,3)$} \\
\hline & & $\left|u\left(t_{M}\right)-u_{M}\right|$ & Rate & $\left|u\left(t_{M}\right)-u_{M}\right|$ & Rate & $\left|u\left(t_{M}\right)-u_{M}\right|$ & Rate \\
\hline \multirow[t]{5}{*}{0.1} & 32 & $8.71434 \mathrm{E}-09$ & - & $4.79960 \mathrm{E}-09$ & - & $7.47072 \mathrm{E}-09$ & - \\
\hline & 64 & $6.27059 \mathrm{E}-10$ & 3.80 & $2.95994 \mathrm{E}-10$ & 4.02 & $5.39926 \mathrm{E}-10$ & 3.79 \\
\hline & 128 & $4.46205 \mathrm{E}-11$ & 3.81 & $1.81797 \mathrm{E}-11$ & 4.03 & $3.86592 \mathrm{E}-11$ & 3.80 \\
\hline & 256 & $3.15064 \mathrm{E}-12$ & 3.82 & $1.11308 \mathrm{E}-12$ & 4.03 & $2.74569 \mathrm{E}-12$ & 3.82 \\
\hline & 512 & $2.15830 \mathrm{E}-13$ & 3.87 & $6.89707 \mathrm{E}-14$ & 4.01 & $1.94223 \mathrm{E}-13$ & 3.82 \\
\hline \multirow[t]{5}{*}{0.3} & 32 & $5.23976 \mathrm{E}-08$ & - & $3.73908 \mathrm{E}-08$ & - & $4.68436 \mathrm{E}-08$ & - \\
\hline & 64 & $4.16942 \mathrm{E}-09$ & 3.65 & $2.78549 \mathrm{E}-09$ & 3.75 & $3.72285 \mathrm{E}-09$ & 3.65 \\
\hline & 128 & $3.28853 \mathrm{E}-10$ & 3.66 & $2.08205 \mathrm{E}-10$ & 3.74 & $2.93824 \mathrm{E}-10$ & 3.66 \\
\hline & 256 & $2.57840 \mathrm{E}-11$ & 3.67 & $1.56249 \mathrm{E}-11$ & 3.74 & $2.30683 \mathrm{E}-11$ & 3.67 \\
\hline & 512 & $2.01141 \mathrm{E}-12$ & 3.68 & $1.17766 \mathrm{E}-12$ & 3.73 & $1.80270 \mathrm{E}-12$ & 3.68 \\
\hline \multirow[t]{5}{*}{0.5} & 32 & $1.90898 \mathrm{E}-07$ & - & $1.62656 \mathrm{E}-07$ & - & $1.78745 \mathrm{E}-07$ & - \\
\hline & 64 & $1.71443 \mathrm{E}-08$ & 3.48 & $1.42466 \mathrm{E}-08$ & 3.51 & $1.60163 \mathrm{E}-08$ & 3.48 \\
\hline & 128 & $1.53023 \mathrm{E}-09$ & 3.49 & $1.24931 \mathrm{E}-09$ & 3.51 & $1.42842 \mathrm{E}-09$ & 3.49 \\
\hline & 256 & $1.36106 \mathrm{E}-10$ & 3.49 & $1.09734 \mathrm{E}-10$ & 3.51 & $1.27023 \mathrm{E}-10$ & 3.49 \\
\hline & 512 & $1.20810 \mathrm{E}-11$ & 3.49 & $9.65029 \mathrm{E}-12$ & 3.51 & $1.12707 \mathrm{E}-11$ & 3.49 \\
\hline \multirow[t]{5}{*}{0.7} & 32 & $6.21914 \mathrm{E}-07$ & - & $5.86156 \mathrm{E}-07$ & - & $6.04267 \mathrm{E}-07$ & - \\
\hline & 64 & $6.37330 \mathrm{E}-08$ & 3.29 & $5.95612 \mathrm{E}-08$ & 3.30 & $6.18239 \mathrm{E}-08$ & 3.29 \\
\hline & 128 & $6.50169 \mathrm{E}-09$ & 3.29 & $6.04415 \mathrm{E}-09$ & 3.30 & $6.30254 \mathrm{E}-09$ & 3.29 \\
\hline & 256 & $6.61800 \mathrm{E}-10$ & 3.30 & $6.13212 \mathrm{E}-10$ & 3.30 & $6.41338 \mathrm{E}-10$ & 3.30 \\
\hline & 512 & $6.72836 \mathrm{E}-11$ & 3.30 & $6.22274 \mathrm{E}-11$ & 3.30 & $6.52096 \mathrm{E}-11$ & 3.30 \\
\hline
\end{tabular}

Acknowledgements The authors would like to thank Jason Frank and WenYi Tian for helpful discussions. Han Zhou also thanks the support from China Scholarship Council (CSC).

Open Access This article is distributed under the terms of the Creative Commons Attribution 4.0 International License (http://creativecommons.org/licenses/by/4.0/), which permits unrestricted use, distribution, and reproduction in any medium, provided you give appropriate credit to the original author(s) and the source, provide a link to the Creative Commons license, and indicate if changes were made. 


\section{A Preliminary Results for Theorems 3.1 and 3.2}

Lemma A.1 Let $k \in \mathbb{N}$ and $n \geq k . I_{n, q}^{r}$ is defined by (2.13). Then it holds that

$$
(-1)^{k+r+1} \nabla^{k} I_{n, q}^{r} \geq 0,
$$

if $r \leq q$. Otherwise,

$$
(-1)^{k+q+1} \nabla^{k} I_{n, q}^{r} \geq 0 .
$$

Proof First, if $r \leq q$, from (2.13), we have

$$
\begin{aligned}
I_{n, q}^{r} & =\frac{1}{\Gamma(1-\alpha)} \int_{0}^{1}(n+1-s)^{-\alpha} \mathrm{d}\left(\begin{array}{c}
s-q+r-1 \\
r
\end{array}\right) \\
& =\frac{1}{\Gamma(1-\alpha)} \int_{0}^{1}(n+1-s)^{-\alpha} \sum_{n=0}^{r-1} \frac{1}{(s-q+n)}\left(\begin{array}{c}
s-q+r-1 \\
r
\end{array}\right) \mathrm{d} s .
\end{aligned}
$$

Since $(s-q+n) \leq 0$ for $0 \leq s \leq 1$ and $n=0, \ldots, r-1$, we obtain $(-1)^{r}\left(\begin{array}{c}s-q+r-1 \\ r\end{array}\right) \geq 0$ and $(-1)^{r+1} \frac{\mathrm{d}}{\mathrm{d} s}\left(\begin{array}{c}s-q+r-1 \\ r\end{array}\right) \geq 0$. This leads to $(-1)^{r+1} I_{n, q}^{r} \geq 0$. In addition, we can see that

$$
\begin{aligned}
\nabla I_{n, q}^{r} & =\frac{1}{\Gamma(1-\alpha)} \int_{0}^{1}\left((n+1-s)^{-\alpha}-(n-s)^{-\alpha}\right) \mathrm{d}\left(\begin{array}{c}
s-q+r-1 \\
r
\end{array}\right) \\
& =\frac{-\alpha}{\Gamma(1-\alpha)} \int_{0}^{1} \int_{n}^{n+1}(\xi-s)^{-\alpha-1} \mathrm{~d} \xi \mathrm{d}\left(\begin{array}{c}
s-q+r-1 \\
r
\end{array}\right) \\
& =\frac{-\alpha}{\Gamma(1-\alpha)} \int_{0}^{1} \int_{0}^{1}(\xi+n-s)^{-\alpha-1} \mathrm{~d} \xi \mathrm{d}\left(\begin{array}{c}
s-q+r-1 \\
r
\end{array}\right),
\end{aligned}
$$

where $(\xi+n-s)^{-\alpha-1} \geq 0$ for $n \geq 1$ and $0 \leq \xi, s \leq 1$, then $(-1)^{r+2} \nabla I_{n, q}^{r} \geq 0$.

For $k \geq 2$, assume that

$$
\nabla^{k-1} I_{n, q}^{r}=\frac{(-\alpha)_{k-1}}{\Gamma(1-\alpha)} \int_{[0,1]^{k}}\left(\sum_{i=1}^{k-1} \xi_{i}+n-k+2-s\right)^{-\alpha-k+1} \mathrm{~d}^{k-1} \xi \mathrm{d}\left(\begin{array}{c}
s-q+r-1 \\
r
\end{array}\right)
$$

where $(\alpha)_{k-1}=\alpha(\alpha-1) \cdots(\alpha-k+2)$ and $\mathrm{d}^{k-1} \xi=\mathrm{d} \xi_{1} \cdots \mathrm{d} \xi_{k-1}$. Then

$$
\begin{aligned}
\nabla^{k} I_{n, q}^{r} & =\nabla^{k-1} I_{n, q}^{r}-\nabla^{k-1} I_{n-1, q}^{r} \\
& =\frac{(-\alpha)_{k-1}}{\Gamma(1-\alpha)} \int_{[0,1]^{k}} \nabla\left(\sum_{i=1}^{k-1} \xi_{i}+n-k+2-s\right)^{-\alpha-k+1} \mathrm{~d}^{k-1} \xi \mathrm{d}\left(\begin{array}{c}
s-q+r-1 \\
r
\end{array}\right) \\
& =\frac{(-\alpha)_{k}}{\Gamma(1-\alpha)} \int_{[0,1]^{k}} \int_{n+1}^{n+2}\left(\sum_{i=1}^{k} \xi_{i}-k-s\right)^{-\alpha-k} \mathrm{~d} \xi_{k} \mathrm{~d}^{k-1} \xi \mathrm{d}\left(\begin{array}{c}
s-q+r-1 \\
r
\end{array}\right) \\
& =\frac{(-\alpha)_{k}}{\Gamma(1-\alpha)} \int_{[0,1]^{k+1}}\left(\sum_{i=1}^{k} \xi_{i}+n-k+1-s\right)^{-\alpha-k} \mathrm{~d}^{k} \xi \mathrm{d}\left(\begin{array}{c}
s-r+1 \\
r
\end{array}\right) .
\end{aligned}
$$

Since $\left(\sum_{i=1}^{k} \xi_{i}+n-k+1-s\right) \geq 0$ for $n \geq k \geq 1$ and $0 \leq \xi_{i}, s \leq 1$, we can get the result (A.1). 
In the case $r \geq q+1$, by means of integrating by part, we get

$$
\begin{aligned}
I_{n, q}^{r} & =\frac{1}{\Gamma(1-\alpha)} \int_{0}^{1}(n+1-s)^{-\alpha} \mathrm{d}\left(\begin{array}{c}
s-q+r-1 \\
r
\end{array}\right) \\
& =\frac{-\alpha}{\Gamma(1-\alpha)} \int_{0}^{1}(n+1-s)^{-\alpha-1}\left(\begin{array}{c}
s-q+r-1 \\
r
\end{array}\right) \mathrm{d} s .
\end{aligned}
$$

Since $\left(\begin{array}{c}s-q+r-1 \\ r\end{array}\right)$ includes a factor $s(s-1)$ for $r \geq q+1, q \in \mathbb{N}^{+}$, the sign of $\left(\begin{array}{c}s-q+r-1 \\ r\end{array}\right)$ equals the sign of $\prod_{i=1}^{q}(s-i)$. Thus, from $(-1)^{q}\left(\begin{array}{c}s-q+r-1 \\ r\end{array}\right) \geq 0$ it follows that $(-1)^{q+1} I_{n, q}^{r} \geq 0$ for $n \geq 0$. Furthermore, an induction process yields

$$
\nabla^{k} I_{n, q}^{r}=\frac{(-\alpha)_{k+1}}{\Gamma(1-\alpha)} \int_{[0,1]^{k+1}}\left(\sum_{i=1}^{k} \xi_{i}+n-k+1-s\right)^{-\alpha-k-1}\left(\begin{array}{c}
s-q+r-1 \\
r
\end{array}\right) \mathrm{d}^{k} \xi \mathrm{d} s
$$

for $n \geq k \geq 1$, which proves (A.2).

Lemma A.2 Set

$$
s_{n}=\frac{1}{\Gamma(1-\alpha)} \int_{0}^{1}(n+1-s)^{-\alpha} \varphi(s) \mathrm{d} s, \quad n \geq 0,
$$

where $\varphi(s) \geq 0$ for $0 \leq s \leq 1$. Then for $n \geq k$, it holds that $(-1)^{k} \nabla^{k} s_{n} \geq 0$.

Proof It is easy to verify that $s_{n} \geq 0$ for all $n \geq 0$, since for $n \geq 0,0 \leq s \leq 1$, one has $(n+1-s)^{-\alpha}>0$ and $\varphi(s) \geq 0$. The definition of $s_{n}$ implies that

$$
\begin{aligned}
\nabla s_{n} & =\frac{1}{\Gamma(1-\alpha)} \int_{0}^{1}\left((n+1-s)^{-\alpha}-(n-s)^{-\alpha}\right) \varphi(s) \mathrm{d} s \\
& =\frac{-\alpha}{\Gamma(1-\alpha)} \int_{0}^{1} \int_{0}^{1}(n+\xi-s)^{-\alpha-1} \varphi(s) \mathrm{d} \xi \mathrm{d} s, \quad n \geq 1 .
\end{aligned}
$$

Since $(n+\xi-s)^{-\alpha-1}>0$ and $\varphi(s) \geq 0$ for $n \geq 1$ and $0 \leq s, \xi \leq 1$, we obtain $\nabla s_{n} \leq 0$. Furthermore, an induction process yields

$$
\nabla^{k} s_{n}=\frac{(-\alpha)_{n}}{\Gamma(1-\alpha)} \int_{[0,1]^{k+1}}\left(\sum_{i=1}^{k} \xi_{i}+n-k+1-s\right)^{-\alpha-k} \varphi(s) \mathrm{d}^{k} \xi \mathrm{d} s
$$

then we can see $(-1)^{k} \nabla^{k} s_{n} \geq 0$ for $n \geq k$.

Lemma A.3 ([31]) Assume that the coefficient sequence of a series a $(\xi)$ is in $l^{1}$. Let $\left|\xi_{0}\right| \leq 1$. Then the coefficient sequence of

$$
b(\xi)=\frac{a(\xi)-a\left(\xi_{0}\right)}{\xi-\xi_{0}}
$$

converges to zero.

Theorem A.1 ([37,43]) Suppose that

$$
f(z)=\sum_{n=0}^{\infty} c_{n} z^{n}, \quad \sum_{n=0}^{\infty}\left|c_{n}\right|<\infty
$$


and $f(z) \neq 0$ for every $|z| \leq 1$. Then

$$
\frac{1}{f(z)}=\sum_{n=0}^{\infty} a_{n} z^{n} \text { with } \sum_{n=0}^{\infty}\left|a_{n}\right|<\infty .
$$

Theorem A.2 ([3,41]) The moment problem

$$
s_{k}=\int_{0}^{1} u^{k} \mathrm{~d} \sigma(u), \quad k=0,1, \ldots
$$

is solvable within the class of non-decreasing functions iff the inequalities

$$
(-1)^{m} \nabla^{m} s_{k} \geq 0
$$

hold for $k \geq m$.

Lemma A.4 Assume that $k \in \mathbb{N}^{+}$and $1 \leq i \leq k$. Then for each pair $(k, i)$, the coefficient sequence of $g^{(k, i)}(\xi)$ in (3.5) converges to zero.

Proof From the expression of $\nabla^{m} I_{n, q}^{r}$ shown in Lemma A.1, we have

$$
\begin{aligned}
& \lim _{n \rightarrow \infty} \nabla^{m} I_{n, q}^{r} \\
& =\frac{(-\alpha)_{m}}{\Gamma(1-\alpha)} \int_{[0,1]^{m+1}} \lim _{n \rightarrow \infty}\left(\sum_{i=1}^{m} \xi_{i}+n-m+1-s\right)^{-\alpha-m} \mathrm{~d}^{m} \xi \mathrm{d}\left(\begin{array}{c}
s-q+r-1 \\
r
\end{array}\right) \\
& =0
\end{aligned}
$$

or

$$
\begin{aligned}
& \lim _{n \rightarrow \infty} \nabla^{m} I_{n, q}^{r} \\
& =\frac{(-\alpha)_{m+1}}{\Gamma(1-\alpha)} \int_{[0,1]^{m+1}} \lim _{n \rightarrow \infty}\left(\sum_{i=1}^{m} \xi_{i}+n-m+1-s\right)^{-\alpha-m-1}\left(\begin{array}{c}
s-q+r-1 \\
r
\end{array}\right) \mathrm{d}^{m} \xi \mathrm{d} s \\
& =0
\end{aligned}
$$

for some $m, q, r \in \mathbb{N}^{+}$independent of $n$ and $\alpha>0$. Note that $g_{n}^{(k, i)}=-\sum_{j=0}^{k-1} u_{j}\left(w_{n+k, j}^{(k, i)}+\right.$ $\left.\omega_{n+k-j}^{(k, i)}\right)$ is a finite linear combination of $\nabla^{m} I_{n+l, q}^{r}$ with $0 \leq m \leq k$ if $k$ is finite. This gives $g_{n}^{(k, i)} \rightarrow 0$ as $n \rightarrow \infty$ for bounded $\left\{u_{j}\right\}_{j=0}^{k-1}$.

Lemma A.5 For $1 \leq i \leq k \leq 6$, the coefficient sequence of $\omega^{(k, i)}(\xi)$ belongs to $l^{1}$ space.

Proof As indicated in Lemma A.1 and Lemma A.4, the following relation

$$
\sum_{n=p}^{\infty}\left|\nabla^{k} I_{n, q}^{r}\right|=\left|\sum_{n=p}^{\infty}\left(\nabla^{k-1} I_{n, q}^{r}-\nabla^{k-1} I_{n-1, q}^{r}\right)\right|=\left|\nabla^{k-1} I_{p-1, q}^{r}\right|
$$

holds for $p \geq k \geq 1$. Therefore, by the definition of $\left\{\omega_{n}^{(k, i)}\right\}_{n=0}^{\infty}$, we can find finite positive integers $M=M(k, i)$ such that 


$$
\begin{aligned}
\sum_{n=0}^{\infty}\left|\omega_{n}^{(k, i)}\right| & \leq \sum_{n=0}^{M}\left|\omega_{n}^{(k, i)}\right|+\sum_{m=1}^{k} \sum_{n=m}^{\infty}\left|\nabla^{m} I_{n, i}^{m}\right| \\
& \leq \sum_{n=0}^{M}\left|\omega_{n}^{(k, i)}\right|+\sum_{m=1}^{k}\left|\nabla^{m-1} I_{m-1, i}^{m}\right|,
\end{aligned}
$$

which leads to the result.

Lemma A.6 For $1 \leq i \leq k \leq 6$ and $\left|\xi_{0}\right| \leq 1$, the coefficient sequence of (1 $\xi) \frac{\omega^{(k, i)}(\xi)-\omega^{(k, i)}\left(\xi_{0}\right)}{\xi-\xi_{0}}$ belongs to the space $l^{1}$.

Proof Using the expression of $\omega^{(k, i)}(\xi)$, we have

$$
\begin{aligned}
(1-\xi) \frac{\omega^{(k, i)}(\xi)-\omega^{(k, i)}\left(\xi_{0}\right)}{\xi-\xi_{0}} & =(1-\xi) \sum_{n=0}^{\infty} \omega_{n}^{(k, i)} \frac{\xi^{n}-\xi_{0}^{n}}{\xi-\xi_{0}} \\
& =(1-\xi) \sum_{n=1}^{\infty} \omega_{n}^{(k, i)} \sum_{m=0}^{n-1} \xi_{0}^{n-1-m} \xi^{m} \\
& =(1-\xi) \sum_{m=0}^{\infty} \sum_{n=0}^{\infty} \omega_{n+m+1}^{(k, i)} \xi_{0}^{n} \xi^{m} \\
& =\sum_{n=0}^{\infty} \omega_{n+1}^{(k, i)} \xi_{0}^{n}+\sum_{m=1}^{\infty}\left(\sum_{n=0}^{\infty} \nabla \omega_{n+m+1}^{(k, i)} \xi_{0}^{n}\right) \xi^{m}
\end{aligned}
$$

On the one hand, from Lemma A.5, we find

$$
\left|\sum_{n=0}^{\infty} \omega_{n+1}^{(k, i)} \xi_{0}^{n}\right| \leq \sum_{n=0}^{\infty}\left|\omega_{n+1}^{(k, i)} \| \xi_{0}\right|^{n} \leq \sum_{n=0}^{\infty}\left|\omega_{n+1}^{(k, i)}\right|<+\infty .
$$

On the other hand, by the definition of $\left\{\nabla^{k+1} I_{n, q}^{r}\right\}_{n=k+1}^{\infty}$ in Lemma A.1, it can be verified that

$$
\begin{aligned}
\sum_{m=p}^{\infty} \sum_{n=0}^{\infty}\left|\nabla^{k+1} I_{m+n+1, q}^{r}\right| & =\left|\sum_{m=p}^{\infty} \sum_{n=0}^{\infty}\left(\nabla^{k} I_{m+n+1, q}^{r}-\nabla^{k} I_{m+n, q}^{r}\right)\right| \\
& =\left|\sum_{m=p}^{\infty}\left(\nabla^{k-1} I_{m, q}^{r}-\nabla^{k-1} I_{m-1, q}^{r}\right)\right| \\
& =\left|\nabla^{k-1} I_{p-1, q}^{r}\right|
\end{aligned}
$$

for $p \geq k \geq 1$. Therefore, there exist $M_{1}=M_{1}(k, i) \geq 1$ and $M_{2}=M_{2}(k, i) \geq 0$ such that

$$
\begin{aligned}
\sum_{m=1}^{\infty} \sum_{n=0}^{\infty}\left|\nabla \omega_{n+m+1}^{(k, i)}\right| & \leq \sum_{m=1}^{M_{1}} \sum_{n=0}^{M_{2}}\left|\nabla \omega_{n+m+1}^{(k, i)}\right|+\sum_{p=1}^{k} \sum_{m=p}^{\infty} \sum_{n=0}^{\infty}\left|\nabla^{p+1} I_{m+n+1, i}^{p}\right| \\
& \leq \sum_{m=1}^{M_{1}} \sum_{n=0}^{M_{2}}\left|\nabla \omega_{n+m+1}^{(k, i)}\right|+\sum_{p=1}^{k}\left|\nabla^{p-1} I_{p-1, i}^{p}\right| .
\end{aligned}
$$


Combining this with

$$
\left|\sum_{n=0}^{\infty} \omega_{n+1}^{(k, i)} \xi_{0}^{n}\right|+\sum_{m=1}^{\infty}\left|\sum_{n=0}^{\infty} \nabla \omega_{n+m+1}^{(k, i)} \xi_{0}^{n}\right| \leq \sum_{n=0}^{\infty}\left|\omega_{n+1}^{(k, i)}\right|+\sum_{m=1}^{\infty} \sum_{n=0}^{\infty}\left|\nabla \omega_{n+m+1}^{(k, i)}\right|,
$$

we arrive at the conclusion.

Corollary A.3 For $\left|\xi_{0}\right| \leq 1$ and $1 \leq i \leq k \leq 6$, the sequence

$$
(1-\xi)\left(1-\xi_{0}\right) \frac{\varphi^{(k, i)}(\xi)-\varphi^{(k, i)}\left(\xi_{0}\right)}{\xi-\xi_{0}}
$$

belongs to $l^{1}$, where the series $\varphi^{(k, i)}(\xi)$ satisfy $\omega^{(k, i)}(\xi)=(1-\xi) \varphi^{(k, i)}(\xi)$.

Proof Based on the definition of $\varphi^{(k, i)}(\xi)$, we obtain

$$
\begin{aligned}
(1-\xi) \frac{\omega^{(k, i)}(\xi)-\omega^{(k, i)}\left(\xi_{0}\right)}{\xi-\xi_{0}} & =(1-\xi) \frac{(1-\xi) \varphi^{(k, i)}(\xi)-\left(1-\xi_{0}\right) \varphi^{(k, i)}\left(\xi_{0}\right)}{\xi-\xi_{0}} \\
& =(1-\xi)\left(1-\xi_{0}\right) \frac{\varphi^{(k, i)}(\xi)-\varphi^{(k, i)}\left(\xi_{0}\right)}{\xi-\xi_{0}}-\omega^{(k, i)}(\xi) .
\end{aligned}
$$

From the absolute convergence of the sequences $(1-\xi) \frac{\omega^{(k, i)}(\xi)-\omega^{(k, i)}\left(\xi_{0}\right)}{\xi-\xi_{0}}$ and $\omega^{(k, i)}(\xi)$ given in Lemma A.5 and Lemma A.6, respectively, we arrive at the result.

\section{B Preliminary Results for Theorem 4.1}

Theorem B.1 For $1 \leq i \leq k \leq 3, \omega^{(k, i)}(\xi)$ satisfies the following conclusions:

(i) $\omega^{(k, i)}(\xi) \neq 0$ for $|\xi| \leq 1$ and $\xi \neq 1$;

(ii) $\xi=1$ is a simple zero.

Proof It can be easily verified that $\omega^{(k, i)}(1)=0$, implying that $\xi=1$ is a zero. We rewrite $\omega^{(k, i)}(\xi)$ as

$$
\omega^{(k, i)}(\xi)=(1-\xi) \varphi^{(k, i)}(\xi),
$$

then the proof of results (i) and (ii) is equivalent to proving $\varphi^{(k, i)}(\xi) \neq 0$ for $|\xi| \leq 1$. In the following discussion, we consider three situations of $\xi$ separately, i.e., $|\xi|<1 ;|\xi|=1$ and $\xi \neq 1 ; \xi=1$. First, we prove the results for different $k$ and $i$ respectively under the condition $|\xi|<1$.

Case $|\xi|<1$ : Denote $\xi=|\xi| e^{i \theta}$. In the case $(k, i)=(1,1)$, from (3.7) and (3.8), we deduce

$$
\varphi^{(1,1)}(\xi)=I(\xi)=\int_{0}^{1} \frac{1}{1-r \xi} \mathrm{d} \sigma(\mathrm{r})
$$

furthermore,

$$
\operatorname{Re}\left(\varphi^{(1,1)}(\xi)\right)=\int_{0}^{1} \operatorname{Re}\left(\frac{1}{1-r \xi}\right) \mathrm{d} \sigma(\mathrm{r}):=\int_{0}^{1} f(r,|\xi|, \theta) \mathrm{d} \sigma(\mathrm{r}),
$$

where $f(r,|\xi|, \theta)=\frac{1-r|\xi| \cos \theta}{1-2 r|\xi| \cos \theta+r^{2}|\xi|^{2}}$. In addition,

$$
\frac{\partial f}{\partial \theta}(r,|\xi|, \theta)=\frac{-r|\xi| \sin \theta\left(1-r^{2}|\xi|^{2}\right)}{\left(1-2 r|\xi| \cos \theta+r^{2}|\xi|^{2}\right)^{2}}
$$


for $0 \leq r \leq 1$ and $0 \leq|\xi|<1$ implies that $0<f(r,|\xi|, \pi) \leq f(r,|\xi|, \theta) \leq f(r,|\xi|, 0)$. Thus

$$
\operatorname{Re}\left(\varphi^{(1,1)}(\xi)\right) \geq \int_{0}^{1} \frac{1}{1+r|\xi|} \mathrm{d} \sigma(\mathrm{r}) \geq \frac{I_{0}}{2} .
$$

In the case $(k, i)=(2,1)$, using (3.9), (3.10) and (3.11), we obtain

$$
\begin{aligned}
\varphi^{(2,1)}(\xi) & =I(\xi)-2 I_{1}^{2}(\xi)+(3-\xi) I_{1}^{2}(\xi) \\
& =\int_{0}^{1} \frac{1}{1-r \xi} \mathrm{d} v(r)+\int_{0}^{1} \frac{3-\xi}{1-r \xi} \mathrm{d} \gamma(r),
\end{aligned}
$$

therefore,

$$
\begin{aligned}
\operatorname{Re}\left(\varphi^{(2,1)}(\xi)\right) & =\int_{0}^{1} \operatorname{Re}\left(\frac{1}{1-r \xi}\right) \mathrm{d} v(r)+\int_{0}^{1} \operatorname{Re}\left(\frac{3-\xi}{1-r \xi}\right) \mathrm{d} \gamma(r) \\
& \geq \int_{0}^{1} \frac{1}{1+r|\xi|} \mathrm{d} v(r)+2 \int_{0}^{1} \frac{1}{1+r|\xi|} \mathrm{d} \gamma(r)+\int_{0}^{1} \operatorname{Re}\left(\frac{1-\xi}{1-r \xi}\right) \mathrm{d} \gamma(r) \\
& >\frac{I_{0}}{2} .
\end{aligned}
$$

In the case $\underline{(k, i)=(2,2)}$, it follows from (3.12) that

$$
\varphi^{(2,2)}(\xi)=I_{0,1}^{2}(3-\xi)+(1+\xi) \sum_{n=0}^{\infty} I_{n+1,1}^{2} \xi^{n}+I(\xi)-2 I_{1}^{2}(\xi) .
$$

Consequently,

$$
\begin{aligned}
\operatorname{Re}\left(\varphi^{(2,2)}(\xi)\right) & =I_{0,1}^{2} \operatorname{Re}(3-\xi)+\int_{0}^{1} r \operatorname{Re}\left(\frac{1+\xi}{1-r \xi}\right) \mathrm{d} \gamma(r)+\int_{0}^{1} \operatorname{Re}\left(\frac{1}{1-r \xi}\right) \mathrm{d} v(r) \\
& \geq \frac{I_{0}}{2}+I_{0,1}^{2} .
\end{aligned}
$$

Let

$$
f(r,|\xi|, \theta)=\operatorname{Re}\left(\frac{1+\xi}{1-r \xi}\right)=\frac{1-r|\xi| \cos \theta+|\xi| \cos \theta-r|\xi|^{2}}{1-2 r|\xi| \cos \theta+r^{2}|\xi|^{2}}
$$

Then $\frac{\partial f}{\partial \theta}(r,|\xi|, \theta)=\frac{-|\xi| \sin \theta(r+1)\left(1-r^{2}|\xi|^{2}\right)}{\left(1-2 r|\xi| \cos \theta+r^{2}|\xi|^{2}\right)^{2}}$ implies $f(r,|\xi|, \theta) \geq f(r,|\xi|, \pi)>0$ for $0 \leq$ $r \leq 1$ and $0 \leq|\xi|<1$. This means $\int_{0}^{1} r \operatorname{Re}\left(\frac{1+\xi}{1-r \xi}\right) \mathrm{d} \gamma(r) \geq 0$. In addition, we find $I_{0,1}^{2} \operatorname{Re}(3-\xi) \geq 2 I_{0,1}^{2}$ for $|\xi| \leq 1$. Therefore, we obtain $\operatorname{Re}\left(\varphi^{(2,2)}(\xi)\right) \geq \frac{I_{0}}{2}+I_{0,1}^{2}>0$.

In the case $(k, i)=(3,1)$, using $(2.14)$, we have

$$
\omega^{(3,1)}(\xi)=(1-\xi) I(\xi)+(1-\xi)^{2} I_{1}^{2}(\xi)+(1-\xi)^{3} I_{1}^{3}(\xi),
$$

and consequently,

$$
\begin{aligned}
\varphi^{(3,1)}(\xi) & =I(\xi)+(1-\xi) I_{1}^{2}(\xi)+(1-\xi)^{2} I_{1}^{3}(\xi) \\
& =I(\xi)-3 I_{1}^{3}(\xi)+(1-\xi) I_{1}^{2}(\xi)+\left(4-2 \xi+\xi^{2}\right) I_{1}^{3}(\xi)
\end{aligned}
$$

Lemma A.2 and Theorem A.2 yield the existence of a non-decreasing function $\eta$ such that

$$
I_{n}-3 I_{n, 1}^{3}=\int_{0}^{1} r^{n} \mathrm{~d} \eta(r), \quad n=0,1, \ldots
$$


From Lemma A.1, we also obtain

$$
I_{n, 1}^{3}=\int_{0}^{1} r^{n} \mathrm{~d} \beta(r), \quad n=0,1, \ldots,
$$

where $\beta(r)$ is non-decreasing on $[0,1]$. Thus,

$$
\begin{aligned}
\operatorname{Re}\left(\varphi^{(3,1)}(\xi)\right)= & \int_{0}^{1} \operatorname{Re}\left(\frac{1}{1-r \xi}\right) \mathrm{d} \eta(r)+\int_{0}^{1} \operatorname{Re}\left(\frac{1-\xi}{1-r \xi}\right) \mathrm{d} \gamma(r) \\
& +\int_{0}^{1} \operatorname{Re}\left(\frac{4-2 \xi+\xi^{2}}{1-r \xi}\right) \mathrm{d} \beta(r) .
\end{aligned}
$$

Since

$$
\begin{aligned}
& \operatorname{Re}\left(\frac{\frac{3}{2}-2 \xi+\xi^{2}}{1-r \xi}\right) \\
& =\frac{(1-r|\xi| \cos \theta)\left(\frac{3}{2}-2|\xi| \cos \theta+|\xi|^{2} \cos 2 \theta\right)+2 r|\xi|^{2} \sin ^{2} \theta(1-|\xi| \cos \theta)}{1-2 r|\xi| \cos \theta+r^{2}|\xi|^{2}},
\end{aligned}
$$

and

$$
\frac{3}{2}-2|\xi| \cos \theta+|\xi|^{2} \cos 2 \theta=\frac{1}{2}(1-2|\xi| \cos \theta)^{2}+\left(1-|\xi|^{2}\right) \geq 0
$$

for $|\xi| \leq 1$ and $\theta \in \mathbb{R}$, we have

$$
\operatorname{Re}\left(\varphi^{(3,1)}(\xi)\right) \geq \int_{0}^{1} \frac{1}{1+r|\xi|} \mathrm{d} \eta(r)+\frac{5}{2} \int_{0}^{1} \frac{1}{1+r|\xi|} \mathrm{d} \beta(r) \geq \frac{1}{2} I_{0}-\frac{1}{4} I_{0,1}^{3} .
$$

In the case $(k, i)=(3,2)$, by $(2.15)$, we derive

$$
\begin{aligned}
\varphi^{(3,2)}(\xi)= & I_{0}+\sum_{j=0}^{\infty} I_{j+1} \xi^{j}+I_{0,1}^{2}(1-\xi)+(1-\xi) \sum_{j=0}^{\infty} I_{j+1,2}^{2} \xi^{j}+(1-\xi)^{2} I_{0,1}^{3} \\
& +(1-\xi)^{2} \sum_{j=0}^{\infty} I_{j+1,2}^{3} \xi^{j}
\end{aligned}
$$

Substituting the relations $I_{n, 2}^{2}=I_{n, 1}^{2}-I_{n}$ and $I_{n, 2}^{3}=I_{n, 1}^{3}-I_{n, 1}^{2}$ into (B.3), we find

$$
\begin{aligned}
\varphi^{(3,2)}(\xi)= & I(\xi)+(1-\xi) I_{1}^{2}(\xi)+\left(1-\xi^{2}\right) \sum_{j=0}^{\infty} I_{j+1,1}^{3} \xi^{j} \\
& -2(1-\xi) I_{1}^{3}(\xi)+(3-\xi)(1-\xi) I_{0,1}^{3} \\
= & \left(\frac{5}{6}+\frac{1}{6} \xi\right) I(\xi)+(1-\xi)\left(I_{1}^{2}(\xi)-2 I_{1}^{3}(\xi)+\frac{1}{6} I(\xi)\right) \\
& +\left(1-\xi^{2}\right) \sum_{j=0}^{\infty} I_{j+1,1}^{3} \xi^{j}+(3-\xi)(1-\xi) I_{0,1}^{3} .
\end{aligned}
$$

Since

$$
I_{n, 1}^{2}-2 I_{n, 1}^{3}+\frac{1}{6} I_{n}=\frac{1}{\Gamma(1-\alpha)} \int_{0}^{1}(n+1-s)^{-\alpha} s(1-s) \mathrm{d} s, \quad n \geq 0,
$$


using Lemma A.2, we obtain

$$
I_{n, 1}^{2}-2 I_{n, 1}^{3}+\frac{1}{6} I_{n}=\int_{0}^{1} r^{n} \mathrm{~d} \mu(r), \quad n=0,1, \ldots
$$

with $\mu(r)$ being non-decreasing on $[0,1]$. Therefore, from (3.8), (B.2) and (B.4), it follows that

$$
\begin{aligned}
\operatorname{Re}\left(\varphi^{(3,2)}(\xi)\right)= & \int_{0}^{1} \operatorname{Re}\left(\frac{\frac{5}{6}+\frac{1}{6} \xi}{1-r \xi}\right) \mathrm{d} \sigma(r)+\int_{0}^{1} \operatorname{Re}\left(\frac{1-\xi}{1-r \xi}\right) \mathrm{d} \mu(r) \\
& +\int_{0}^{1} r \operatorname{Re}\left(\frac{1-\xi^{2}}{1-r \xi}\right) \mathrm{d} \beta(r)+\operatorname{Re}((3-\xi)(1-\xi)) I_{0,1}^{3} \\
\geq & \frac{2}{3} \int_{0}^{1} \frac{1}{1+r|\xi|} \mathrm{d} \sigma(r) \geq \frac{I_{0}}{3} .
\end{aligned}
$$

In the case $(k, i)=(3,3)$, from the relation

$$
\begin{aligned}
\omega^{(3,3)}(\xi)= & (1-\xi)\left(I_{0}+I_{1}\right)+(1-\xi) \sum_{j=0}^{\infty} I_{j+2} \xi^{j}+(1-\xi)^{2}\left(I_{0,1}^{2}+I_{1,2}^{2}\right) \\
& +(1-\xi)^{2} \sum_{j=0}^{\infty} I_{j+2,3}^{2} \xi^{j}+(1-\xi)^{3}\left(I_{0,1}^{3}+I_{1,2}^{3}\right)+(1-\xi)^{3} \sum_{j=0}^{\infty} I_{j+2,3}^{3} \xi^{j},
\end{aligned}
$$

it follows that

$$
\begin{aligned}
\varphi^{(3,3)}(\xi)= & I_{0}+I_{1}+\sum_{j=0}^{\infty} I_{j+2} \xi^{j}+(1-\xi)\left(I_{0,1}^{2}+I_{1,2}^{2}\right)+(1-\xi) \sum_{j=0}^{\infty} I_{j+2,3}^{2} \xi^{j} \\
& +(1-\xi)^{2}\left(I_{0,1}^{3}+I_{1,2}^{3}\right)+(1-\xi)^{2} \sum_{j=0}^{\infty} I_{j+2,3}^{3} \xi^{j}
\end{aligned}
$$

In addition, substituting the following relations

$$
\begin{aligned}
& I_{n, 3}^{2}=I_{n, 2}^{2}-I_{n}=I_{n, 1}^{2}-2 I_{n}, \\
& I_{n, 3}^{3}=I_{n, 2}^{3}-I_{n, 2}^{2}=I_{n, 1}^{3}-2 I_{n, 1}^{2}+I_{n}, \quad n \geq 0
\end{aligned}
$$

into (B.6) yields

$$
\begin{aligned}
\varphi^{(3,3)}(\xi)= & I_{0}+I_{1}+\sum_{j=0}^{\infty} I_{j+2} \xi^{j}+(1-\xi)\left(I_{0,1}^{2}+I_{1,1}^{2}-I_{1}\right) \\
& +(1-\xi) \sum_{j=0}^{\infty}\left(I_{j+2,1}^{2}-2 I_{j+2}\right) \xi^{j} \\
& +(1-\xi)^{2}\left(I_{0,1}^{3}+I_{1,1}^{3}-I_{1,1}^{2}\right)+(1-\xi)^{2} \sum_{j=0}^{\infty}\left(I_{j+2,1}^{3}-2 I_{j+2,1}^{2}+I_{j+2}\right) \xi^{j} \\
= & I(\xi)+(1-\xi) I_{1}^{2}(\xi)-2(1-\xi) I_{1}^{3}(\xi)+\left(1-\xi^{2}\right)
\end{aligned}
$$




$$
\begin{aligned}
& \times \sum_{j=0}^{\infty} I_{j+2,2}^{3} \xi^{j}-\left(3-4 \xi+\xi^{2}\right) \sum_{j=0}^{\infty} I_{j+1,2}^{3} \xi^{j} \\
& +(3-\xi)(1-\xi)\left(I_{0,1}^{3}+I_{1,2}^{3}\right)+\left(1-\xi^{2}\right) \sum_{j=0}^{\infty} I_{j+1,1}^{3} \xi^{j}
\end{aligned}
$$

By Lemma A.1, we find that the sequence $\left(-I_{n, 2}^{3}\right)_{n=0}^{\infty}$ is completely monotonic. Thus there exists a non-decreasing function $\vartheta(r)$ defined on $[0,1]$ such that

$$
-I_{n, 2}^{3}=\int_{0}^{1} r^{n} \mathrm{~d} \vartheta(r), \quad n=0,1, \ldots
$$

This yields

$$
\begin{aligned}
& \left(1-\xi^{2}\right) \sum_{j=0}^{\infty} I_{j+2,2}^{3} \xi^{j}-\left(3-4 \xi+\xi^{2}\right) \sum_{j=0}^{\infty} I_{j+1,2}^{3} \xi^{j} \\
& =\int_{0}^{1} \frac{\left(3 r-r^{2}\right)-4 r \xi+\left(r^{2}+r\right) \xi^{2}}{1-r \xi} \mathrm{d} \vartheta(r)
\end{aligned}
$$

for $|\xi|<1$. Since

$$
\begin{aligned}
& \operatorname{Re}\left(\frac{\left(3 r-r^{2}\right)-4 r \xi+\left(r^{2}+r\right) \xi^{2}}{1-r \xi}\right) \\
& \quad=\frac{\left(3 r-r^{2}\right)(1-r|\xi| \cos \theta)+4 r^{2}|\xi|^{2}-4 r|\xi| \cos \theta+\left(r^{2}+r\right)|\xi|^{2} \cos 2 \theta-\left(r^{3}+r^{2}\right)|\xi|^{3} \cos \theta}{1-2 r|\xi| \cos \theta+r^{2}|\xi|^{2}} \\
& :=f(r,|\xi|, \theta) .
\end{aligned}
$$

Taking partial differentiation with respect to $\theta$ yields

$$
\frac{\partial f}{\partial \theta}(r,|\xi|, \theta)=\frac{r|\xi| \sin \theta}{\left(1-2 r|\xi| \cos \theta+r^{2}|\xi|^{2}\right)^{2}} g(r,|\xi|, \theta),
$$

where

$$
\begin{aligned}
g(r,|\xi|, \theta)= & \left(4+3 r-r^{2}-4(r+1)|\xi| \cos \theta+\left(r^{2}+r\right)|\xi|^{2}\right)\left(1-2 r|\xi| \cos \theta+r^{2}|\xi|^{2}\right) \\
& -2\left(\left(3 r-r^{2}\right)(1-r|\xi| \cos \theta)+4 r^{2}|\xi|^{2}-4 r|\xi| \cos \theta\right. \\
& \left.+\left(r^{2}+r\right)|\xi|^{2} \cos 2 \theta-\left(r^{3}+r^{2}\right)|\xi|^{3} \cos \theta\right) .
\end{aligned}
$$

From

$$
\frac{\partial g}{\partial \theta}(r,|\xi|, \theta)=4|\xi| \sin \theta(r+1)\left(1-2 r|\xi| \cos \theta+r^{2}|\xi|^{2}\right)
$$

it follows that $g(r,|\xi|, 0) \leq g(r,|\xi|, \theta) \leq g(r,|\xi|, \pi)$. In addition, we see

$$
\begin{aligned}
g(r,|\xi|, 0)= & \left(4-3 r+r^{2}\right)-4(1+r)|\xi|+\left(7 r+3 r^{2}+3 r^{3}-r^{4}\right)|\xi|^{2} \\
& -4\left(r^{3}+r^{2}\right)|\xi|^{3}+\left(r^{3}+r^{4}\right)|\xi|^{4}
\end{aligned}
$$

and

$$
\begin{aligned}
\frac{\partial g}{\partial|\xi|}(r,|\xi|, 0)= & -4(1+r)+2\left(7 r+3 r^{2}+3 r^{3}-r^{4}\right)|\xi|-12\left(r^{3}+r^{2}\right)|\xi|^{2} \\
& +4\left(r^{3}+r^{4}\right)|\xi|^{3}
\end{aligned}
$$


Since

$$
\frac{\partial^{2} g}{\partial|\xi|^{2}}(r,|\xi|, 0)=r\left(12(r+1)(r|\xi|-1)^{2}+2(1-r)^{3}\right) \geq 0
$$

for $0 \leq r \leq 1$ and $0 \leq|\xi|<1$, we obtain that $\frac{\partial g}{\partial|\xi|}(r,|\xi|, 0)<\frac{\partial g}{\partial|\xi|}(r, 1,0)$. Using (B.9), we find

$$
\frac{\partial g}{\partial|\xi|}(r, 1,0)=2\left(r^{3}-3 r+2\right)(r-1) \leq 0
$$

for $0 \leq r \leq 1$. From this it follows that $\frac{\partial g}{\partial|\xi|}(r,|\xi|, 0)<\frac{\partial g}{\partial|\xi|}(r, 1,0) \leq 0$ for all $0 \leq r \leq 1$ and $0 \leq|\xi|<1$. Then we finally obtain

$$
g(r,|\xi|, 0)>g(r, 1,0)=0, \quad 0 \leq r \leq 1, \quad 0 \leq|\xi|<1 .
$$

Hence, it holds that $g(r,|\xi|, \theta) \geq g(r,|\xi|, 0)>0$ in the cases $0 \leq r \leq 1$ and $0 \leq|\xi|<1$. From formula (B.8), we have $f(r,|\xi|, 0) \leq f(r,|\xi|, \theta) \leq f(r,|\xi|, \pi)$ for all $0 \leq r \leq 1$ and $0 \leq|\xi|<1$. The definition of $f(r,|\xi|, \theta)$ in (B.7) yields

$$
f(r,|\xi|, 0)=\frac{3 r-r^{2}-4 r|\xi|+r^{2}|\xi|^{2}+r|\xi|^{2}}{1-r|\xi|} .
$$

Taking its partial derivative with respect to $|\xi|$, we obtain

$$
\begin{aligned}
\frac{\partial f}{\partial|\xi|}(r,|\xi|, 0) & =\frac{r}{(1-r|\xi|)^{2}}\left(-4+3 r-r^{2}+2(r+1)|\xi|-\left(r^{2}+r\right)|\xi|^{2}\right) \\
& =\frac{r}{(1-r|\xi|)^{2}} h(r,|\xi|) .
\end{aligned}
$$

The inequality $\frac{\partial h}{\partial|\xi|}(r,|\xi|) \geq 2\left(1-r^{2}\right) \geq 0$ for $0 \leq r \leq 1$ with $h(r, 1)=-2(1-$ $r)^{2} \leq 0$ yields $h(r,|\xi|) \leq h(r, 1) \leq 0$ for $0 \leq r \leq 1$. Consequently, in combination with $\frac{\partial f}{\partial|\xi|}(r,|\xi|, 0) \leq 0$, we have $f(r,|\xi|, 0) \geq f(r, 1,0)$ for $0 \leq r \leq 1$ and $0 \leq|\xi|<1$. Further, from $f(r, 1,0)=0$, it follows that

$$
f(r,|\xi|, \theta) \geq f(r,|\xi|, 0) \geq 0, \quad \forall 0 \leq r \leq 1, \quad|\xi|<1, \quad \theta \in \mathbb{R} .
$$

Therefore,

$$
\begin{aligned}
\operatorname{Re}\left(\varphi^{(3,3)}(\xi)\right)= & \int_{0}^{1} \operatorname{Re}\left(\frac{\frac{5}{6}+\frac{1}{6} \xi}{1-r \xi}\right) \mathrm{d} \sigma(r)+\int_{0}^{1} \operatorname{Re}\left(\frac{1-\xi}{1-r \xi}\right) \mathrm{d} \mu(r)+\int_{0}^{1} r \operatorname{Re}\left(\frac{1-\xi^{2}}{1-r \xi}\right) \mathrm{d} \beta(r) \\
& +\int_{0}^{1} \operatorname{Re}\left(\frac{\left(3 r-r^{2}\right)-4 r \xi+\left(r^{2}+r\right) \xi^{2}}{1-r \xi}\right) \mathrm{d} \vartheta(r) \\
& +\operatorname{Re}((3-\xi)(1-\xi))\left(I_{0,1}^{3}+I_{1,2}^{3}\right) \\
\geq & \frac{2}{3} \int_{0}^{1} \frac{1}{1+r|\xi|} \mathrm{d} \sigma(r) \geq \frac{I_{0}}{3}
\end{aligned}
$$

where the last inequality follows from the fact that $I_{0,1}^{3}+I_{1,2}^{3}=\frac{2^{1-\alpha}\left(\alpha^{2}+\alpha\right)}{3 \Gamma(4-\alpha)} \geq 0$ for all $0 \leq \alpha \leq 1$.

Case $\xi=1$ : assume that $\varphi^{(k, i)}(1)=0$, from the definition of $\varphi^{(k, i)}(\xi)$, we find

$$
\varphi^{(k, i)}(\xi)=I(\xi)+l^{(k, i)}(\xi),
$$


where $l^{(k, i)}(\xi)$ are absolutely convergent series. The definition of the coefficients of $I(\xi)$ yields that $\sum_{i=0}^{n} I_{i}$ is arbitrary large as increasing $n$. However, the boundedness of $l^{(k, i)}(1)$ contradicts (B.10) with $\xi=1$, which shows $\varphi^{(k, i)}(1) \neq 0$.

Case $|\xi|=1$ and $\xi \neq 1$ : from Corollary A.3, it follows that $\varphi^{(k, i)}(\xi)$ is pointwise continuous for $|\xi| \leq 1$ except $\xi=1$. If $\xi_{n}=\left(1-\frac{1}{n}\right) \xi$ with $\left|\xi_{n}\right|<1$ for all $n \in \mathbb{N}^{+}$, then $\varphi^{(k, i)}(\xi)$ are the limit points of the sequences $\varphi^{(k, i)}\left(\xi_{n}\right)$. Further, there exist positive constants $c^{(k, i)}$, which are independent of $n$, such that

$$
\operatorname{Re}\left(\varphi^{(k, i)}(\xi)\right)=\lim _{n \rightarrow+\infty} \operatorname{Re}\left(\varphi^{(k, i)}\left(\xi_{n}\right)\right) \geq c^{(k, i)}
$$

Lemma B.1 Let $1 \leq i \leq k \leq 3$ and $0 \leq m \leq k-1,\left\{s_{n, m}^{(k, i)}\right\}_{n=0}^{\infty}$ are defined in (4.6). Then there exist bounded constants $c_{m}^{(k, i)}>0$ independent of $n$ and $\alpha$ such that

$$
\left|s_{n, 0}^{(k, i)}\right| \leq \frac{c_{0}^{(k, i)} n^{-\alpha}}{\Gamma(1-\alpha)}, \quad\left|s_{n, m}^{(k, i)}\right| \leq \frac{c_{m}^{(k, i)} n^{-\alpha-1}}{|\Gamma(-\alpha)|}
$$

for $n \geq 1$ and $m \geq 1$.

Proof It is known from (2.13) that for any finite $q, r \in \mathbb{N}^{+}, I_{n, q}^{r}$ is bounded for $n \in \mathbb{Z}$. Since the coefficients $s_{n, m}^{(k, i)}$ are denoted as the linear combinations of $I_{n, q}^{r}$, we can immediately obtain the boundedness of $s_{n, m}^{(k, i)}$ for all integer $n \geq 0$.

Moreover, for $1 \leq i \leq k \leq 3, s_{n, 0}^{(k, i)}$ can be expressed as a linear combination of $I_{l}$ and $I_{l, 1}^{r}$ with $l \geq n$ and $1 \leq r \leq 3$. Using formulae (A.3) and (A.6), we obtain $I_{n}=O\left(\frac{n^{-\alpha}}{\Gamma(1-\alpha)}\right)$ and $I_{n, 1}^{r}=O\left(\frac{n^{-\alpha-1}}{\Gamma(-\alpha)}\right)=o\left(\frac{n^{-\alpha}}{\Gamma(1-\alpha)}\right)$ for $r \geq 2$ and $n \geq 1$. This implies that there is a uniform bound independent of $n$, denoted by $c_{0}^{(k, i)}>0$, such that $\left|s_{n, 0}^{(k, i)}\right| \leq \frac{c_{0}^{(k, i)} n^{-\alpha}}{\Gamma(1-\alpha)}$ for $n \geq 1$.

In terms of $m \geq 1$, observe that $s_{n, m}^{(k, i)}$ are the linear combinations of $\nabla I_{l}, I_{l, 1}^{r}$ and $\nabla^{p} I_{l, 1}^{r}$ for $l \geq n+1, r \geq 2$ and $1 \leq p \leq 3$. From formulae (A.4) and (A.7), we know that $\nabla I_{n}=O\left(\frac{(n-1)^{-\alpha-1}}{\Gamma(-\alpha)}\right)=O\left(\frac{n^{-\alpha-1}}{\Gamma(-\alpha)}\right)$ and $\nabla^{p} I_{n, 1}^{r}=O\left(\frac{(n-p)^{-\alpha-p-1}}{\Gamma(-\alpha-p+1)}\right)=o\left(\frac{n^{-\alpha-1}}{\Gamma(-\alpha)}\right)$ for $r \geq 2$, therefore we get $s_{n, m}^{(k, i)}=O\left(\frac{n^{-\alpha-1}}{\Gamma(-\alpha)}\right)$, and hence there exist constants $c_{m}^{(k, i)}>0$ such that the last inequality of (B.11) holds.

Lemma B.2 Assume that $\left\{g_{n}^{(\beta)}\right\}_{n=0}^{\infty}$ are generated by the series $(1-\xi)^{\beta}$ for $\beta \in \mathbb{R}$, i.e.,

$$
(1-\xi)^{\beta}=\sum_{n=0}^{\infty}(-1)^{n}\left(\begin{array}{l}
\beta \\
n
\end{array}\right) \xi^{n}=\sum_{n=0}^{\infty} g_{n}^{(\beta)} \xi^{n} .
$$


Then the following relations hold

$$
\begin{cases}\beta \in(-1,0): & g_{0}^{(\beta)}=1, \quad g_{0}^{(\beta)}>g_{1}^{(\beta)}>\cdots>0, \\ & \sum_{i=0}^{n} g_{i}^{(\beta)}=g_{n}^{(\beta-1)}, \quad n \geq 0 \\ \beta \in(0,1): \quad & g_{0}^{(\beta)}=1, \quad g_{n}^{(\beta)}<0, \quad n \geq 1, \\ & 1>\left|g_{1}^{(\beta)}\right|>\left|g_{2}^{(\beta)}\right|>\cdots>0, \\ & \sum_{i=0}^{\infty} g_{i}^{(\beta)}=0, \quad \sum_{i=0}^{n} g_{i}^{(\beta)}=g_{n}^{(\beta-1)}, \quad n \geq 0 .\end{cases}
$$

From (B.1), we set

$$
\omega^{(k, i)}(\xi)=(1-\xi)^{\alpha} \psi^{(k, i)}(\xi)
$$

for $0<\alpha<1$, and

$$
\psi^{(k, i)}(\xi)=(1-\xi)^{1-\alpha} \varphi^{(k, i)}(\xi)
$$

Note that (B.14) implies a relation between the proposed methods and the fractional Euler method mentioned in [30]. In the following part, we discuss some relevant properties of the series $\psi^{(k, i)}(\xi)$.

Lemma B.3 For $1 \leq i \leq k \leq 6$, the coefficient sequence of $\psi^{(k, i)}(\xi)$ belongs to the space $l^{1}$.

Proof Using the expressions of $\varphi^{(k, i)}(\xi)$ presented in Theorem B.1, we obtain

$$
\varphi^{(k, i)}(\xi)=I(\xi)+l^{(k, i)}(\xi), \quad \text { with } \quad \sum_{n=0}^{\infty}\left|l_{n}^{(k, i)}\right|<\infty .
$$

Together with (B.15), it follows that

$$
\psi^{(k, i)}(\xi)=(1-\xi)^{(1-\alpha)} I(\xi)+(1-\xi)^{(1-\alpha)} l^{(k, i)}(\xi) .
$$

Therefore, it suffices to prove that the coefficient sequence of $(1-\xi)^{1-\alpha} I(\xi)$ belongs to $l^{1}$.

From the definition of Gamma function

$$
\Gamma(\beta)=\lim _{n \rightarrow \infty} \frac{n^{\beta}}{(-1)^{n}\left(\begin{array}{c}
-\beta \\
n
\end{array}\right)(n+\beta)}, \quad \beta \neq 0,-1,-2, \ldots,
$$

we obtain the asymptotic relation

$$
\frac{n^{\beta-1}}{\Gamma(\beta)} \cong(-1)^{n}\left(\begin{array}{c}
-\beta \\
n
\end{array}\right), \quad \text { as } n \rightarrow \infty
$$

where the notation $\cong$ means that $\left(n^{\beta-1} / \Gamma(\beta)\right) /(-1)^{n}\left(\begin{array}{c}-\beta \\ n\end{array}\right) \rightarrow 1$ as $n \rightarrow \infty$. Furthermore, it is known from $[16,31]$ that 


$$
(-1)^{n}\left(\begin{array}{c}
-\beta \\
n
\end{array}\right)=\frac{n^{\beta-1}}{\Gamma(\beta)}\left(1+O\left(\frac{\beta-1}{n}\right)\right) .
$$

Also, the definition of $I_{n}$ yields that $I_{n} \cong \frac{n^{-\alpha}}{\Gamma(1-\alpha)}$ as $n \rightarrow \infty$, and

$$
\begin{aligned}
\sum_{n=1}^{\infty}\left|I_{n}-\frac{n^{-\alpha}}{\Gamma(1-\alpha)}\right| & =\frac{1}{\Gamma(1-\alpha)} \sum_{n=1}^{\infty} \int_{0}^{1}\left(n^{-\alpha}-(n+1-s)^{-\alpha}\right) \mathrm{d} s \\
& =\frac{\alpha}{\Gamma(1-\alpha)} \int_{0}^{1} \int_{0}^{1-s} \sum_{n=1}^{\infty}(n+t)^{-\alpha-1} \mathrm{~d} t \mathrm{~d} s \\
& \leq \frac{\alpha}{\Gamma(1-\alpha)} \sum_{n=1}^{\infty} n^{-\alpha-1} \\
& \leq \frac{\alpha}{\Gamma(1-\alpha)}\left(1+\int_{1}^{\infty} x^{-\alpha-1} \mathrm{~d} x\right) \\
& =\frac{\alpha+1}{\Gamma(1-\alpha)}<+\infty .
\end{aligned}
$$

Combine this with (B.18), we get

$$
I_{n}=g_{n}^{(\alpha-1)}+v_{n}, \quad \text { with } \sum_{n=0}^{\infty}\left|v_{n}\right|<\infty .
$$

Hence,

$$
(1-\xi)^{1-\alpha} I(\xi)=\sum_{n=0}^{\infty}\left(\sum_{k=0}^{n} g_{n-k}^{(1-\alpha)} I_{k}\right) \xi^{n}
$$

in combination with the relation

$$
\begin{aligned}
\sum_{n=0}^{\infty}\left|\sum_{k=0}^{n} g_{n-k}^{(1-\alpha)} I_{k}\right| & =\sum_{n=0}^{\infty}\left|\sum_{k=0}^{n} g_{n-k}^{(1-\alpha)}\left(g_{k}^{(\alpha-1)}+v_{k}\right)\right| \\
& \leq \sum_{n=0}^{\infty}\left|\sum_{k=0}^{n} g_{n-k}^{(1-\alpha)} g_{k}^{(\alpha-1)}\right|+\sum_{n=0}^{\infty}\left|\sum_{k=0}^{n} g_{n-k}^{(1-\alpha)} v_{k}\right| \\
& \leq 1+\sum_{n=0}^{\infty}\left|g_{n}^{(1-\alpha)}\right| \sum_{k=0}^{\infty}\left|v_{k}\right|<\infty
\end{aligned}
$$

yields the result.

Lemma B.4 Let $1 \leq i \leq k \leq 3$. Then it holds that $\psi^{(k, i)}(\xi) \neq 0$ for any $|\xi| \leq 1$.

Proof In the proof of Theorem B.1, we know that $\varphi^{(k, i)}(\xi) \neq 0$ for all $|\xi| \leq 1$ and $1 \leq i \leq$ $k \leq 3$. For any $|\xi| \leq 1,(1-\xi)^{1-\alpha}$ is located within the sector $S_{\alpha}=\left\{z:|\arg (z)| \leq \frac{(1-\alpha) \pi}{2}\right\}$. In addition, note that $(1-\xi)^{1-\alpha}=0$ if and only if $\xi=1$. Thus, it remains to find the value of $(1-\xi)^{1-\alpha} \varphi^{(k, i)}(\xi)$ at $\xi=1$. Indeed, from formulae (B.16) and (B.20), we have 


$$
\begin{aligned}
\sum_{n=0}^{\infty} \sum_{l=0}^{n} g_{n-l}^{(1-\alpha)} \varphi_{l}^{(k, i)} & =\sum_{n=0}^{\infty} \sum_{l=0}^{n} g_{n-l}^{(1-\alpha)}\left(g_{l}^{(\alpha-1)}+v_{l}+l_{l}^{(k, i)}\right) \\
& =\sum_{n=0}^{\infty} \sum_{l=0}^{n} g_{n-l}^{(1-\alpha)} g_{l}^{(\alpha-1)}+\sum_{n=0}^{\infty} \sum_{l=0}^{n} g_{n-l}^{(1-\alpha)}\left(v_{l}+l_{l}^{(k, i)}\right) \\
& =1+\sum_{n=0}^{\infty} g_{n}^{(1-\alpha)} \sum_{l=0}^{\infty}\left(v_{l}+l_{l}^{(k, i)}\right)=1,
\end{aligned}
$$

where the last equality holds based on Lemma B.2.

As a result, from Theorem A.1, Lemmas B.3 and B.4, we obtain that for $1 \leq i \leq k \leq 3$ and $0<\alpha<1$, if

$$
\frac{1}{\psi^{(k, i)}(\xi)}=r^{(k, i)}(\xi)=\sum_{n=0}^{\infty} r_{n}^{(k, i)} \xi^{n}
$$

then there exist bounded positive constants $M_{\alpha}^{(k, i)}$, such that $\sum_{n=0}^{\infty}\left|r_{n}^{(k, i)}\right|=M_{\alpha}^{(k, i)}$.

\section{References}

1. Aceto, L., Magherini, C., Novati, P.: Fractional convolution quadrature based on generalized Adams methods. Calcolo 51, 441-463 (2014)

2. Aceto, L., Magherini, C., Novati, P.: On the construction and properties of $m$-step methods for FDEs. SIAM J. Sci. Comput. 37(2), 653-675 (2015)

3. Akhiezer, N.I.: The Classical Moment Problem and Some Related Questions in Analysis. Oliver Boyd, Edinburgh (1965)

4. Alikhanov, A.A.: A new difference scheme for the time fractional diffusion equation. J. Comput. Phys. 280, 424-438 (2015)

5. Bagley, R.L., Torvik, P.J.: A theoretical basis for the application of fractional calculus to viscoelasticity. J. Rheol. 27(3), 201-210 (1983)

6. Baleanu, D., Diethelm, K., Scalas, E.: Fractional Calculus, Models and Numerical Methods, Volume 3 of Series on Complexity, Nonlinearity and Chaos. World Scientific, Singapore (2011)

7. Barkai, E., Metzler, R., Klafter, J.: From continuous time random walks to the fractional Fokker-Planck equation. Phys. Rev. E 61(1), 132-138 (2000)

8. Benson, D.A., Wheatcraft, S.W., Meerschaert, M.M.: Application of a fractional advection-dispersion equation. Water Resour. Res. 36(6), 1403-1412 (2000)

9. Cameron, R.F., Mckee, S.: Product integration methods for second-kind Abel integral equations. J. Comput. Appl. Math. 11(1), 1-10 (1984)

10. Carpinteri, A., Mainardi, F.: Fractals and Fractional Calculus in Continuum Mechanics. Springer, Berlin (1997)

11. de Hoog, F., Weiss, R.: High order methods for a class of Volterra integral equations with weakly singular kernels. SIAM J. Numer. Anal. 11(6), 1166-1180 (1974)

12. Diethelm, K.: The Analysis of Fractional Differential Equations. Lecture Notes in Mathematics. Springer, Berlin (2004)

13. Dixon, J.: On the order of the error in discretization methods for weakly singular second kind non-smooth solutions. BIT Numer. Math. 25(4), 624-634 (1985)

14. Dixon, J., Mckee, S.: Weakly singular discrete Gronwall inequalities. J. Appl. Math. Mech. 66(11), 535-544 (1986)

15. Erdelyi, A.: On fractional integration and its applications to the theory of Hankel transforms. Q. J. Math. 11, 293-303 (1940)

16. Erdelyi, A.: Higher Transcendental Functions, vol. 1. McGraw-Hill, New York (1953)

17. Gao, G.H., Sun, Z.Z., Zhang, H.W.: A new fractional numerical differentiation formula to approximate the Caputo fractional derivative and its applications. J. Comput. Phys. 259, 33-50 (2014) 
18. Garappa, R.: A family of Adams exponential integrators for fractional linear systems. Comput. Math. Appl. 66(5), 717-727 (2013)

19. Hairer, E.: Solving Ordinary Differential Equations II: stiff and Differential-Algebraic Problems, Volume 14 of Springer Series in Computational Mathematics. Springer, Berlin (1991)

20. Herrmann, R.: Fractional Calculus: An Introduction for Physicists, 2nd edn. World Scientific, Singapore (2014)

21. Hilfer, R.: Applications of Fractional Calculus in Physics. World Scientific, Singapore (2000)

22. Kilbas, A.A., Srivastava, H.M., Trujillo, J.J.: Theory and Applications of Fractional Differential Equations, Volume 204 of North-Holland Mathematics Studies. Elsevier, Amsterdam (2006)

23. Langlands, T.A.M., Henry, B.I., Wearne, S.L.: Fractional cable equation models for anomalous electrodiffusion in nerve cells: infinite domain solutions. J. Math. Biol. 59, 761-808 (2009)

24. Li, C., Zeng, F.: Numerical Methods for Fractional Calculus. CRC Press, Taylor \& Francis Group, Boca Raton (2015)

25. Lin, Y.M., Xu, C.J.: Finite difference/spectral approximations for the time-fractional diffusion equation. J. Comput. Phys. 225(2), 1533-1552 (2007)

26. Linz, P.: Numerical methods for Volterra integral equations with singular kernels. SIAM J. Numer. Anal. 6(3), 365-374 (1969)

27. Liu, F., Anh, V., Turner, I., Zhuang, P.: Time fractional advection-dispersion equation. J. Appl. Math. Comput. 13, 233-245 (2003)

28. Lubich, C.: Convolution quadrature and discretized operational calculus. I. Numerische Mathematik 52(2), 129-146 (1988)

29. Lubich, Ch.: Fractional linear multistep methods for Abel-Volterra integral equations of the second kind. Math. Comput. 45(172), 463-469 (1985)

30. Lubich, Ch.: Discretized fractional calculus. SIAM J. Math. Anal. 17(3), 704-719 (1986)

31. Lubich, Ch.: A stability analysis of convolution quadratures for Abel-Volterra integral equations. IMA J. Numer. Anal. 6(6), 87-101 (1986)

32. Lv, C.W., Xu, C.J.: Error analysis of a high order method for time-fractional diffusion equations. SIAM J. Sci. Comput. 38(5), A2699-A2724 (2016)

33. Metzler, R., Klafter, J.: The random walk's guide to anomalous diffusion: a fractional dynamics approach. Phys. Rep. 339(1), 1-77 (2000)

34. Miller, K.S., Ross, B.: An Introduction to Fractional Calculus and Fractional Differential Equations. Wiley, Hoboken (1993)

35. Oldham, K.B., Spanier, J.: The Fractional Calculus. Academic Press, New York (1974)

36. Podlubny, I.: Fractional Differential Equations: An Introduction to Fractional Derivatives, Fractional Differential Equations, to Methods of their Solution and Some of their Applications. Mathematics in Science and Engineering 198. Academic Press, San Diego (1999)

37. Rudin, W.: Real and Complex Analysis. McGraw-Hill, New York (1987)

38. Samko, S.G., Kilbas, A.A., Marichev, O.I.: Fractional Integrals and Derivatives. Gordon and Breach Science Publishers, New York (1993)

39. Santamaria, F., Wils, S., de Schutter, E., Augustine, G.J.: Anomalous diffusion in Purkinje cell dendrites caused by spines. Neuron 52, 635-648 (2006)

40. Scalas, E., Gorenflo, R., Mainardi, F.: Fractional calculus and continuous-time finance. Phys A Stat Mech Appl 284(1-4), 376-384 (2000)

41. Shohat, J.A., Tamarkin, J.D.: The Problem of Moments, Volume 1 of Mathematical Surveys and Monographs, 4th edn. American Mathematical Society, Providence (1970)

42. Wyss, W.: The fractional Black-Scholes equation. Fract. Calc. Appl. Anal. 3, 51-61 (2000)

43. Zygmund, A.: Trigonometric Series. Cambridge University Press, Cambridge (2002) 